\title{
Representations of rank two affine Hecke algebras
}

\author{
Arun Ram* \\ Department of Mathematics \\ Princeton University \\ Princeton, NJ 08544 \\ rama@math.princeton.edu \\ Preprint: August 5, 1998
}

This paper classifies and constructs explicitly all the irreducible representations of affine Hecke algebras of rank two root systems. The methods used to obtain this classification are primarily combinatorial and are, for the most part, an application of the methods used in [Ra1]. I have made special effort to describe how the classification here relates to the classifications by Langlands parameters (coming from $p$-adic group theory) and by indexing triples (coming from a $q$-analogue of the Springer correspondence). There are several reasons for doing the details of this classification:

(a) The proof of the one of the main results of [Ra1] depends on this classification of representations for rank two affine Hecke algebras. Specifically, in the proof of Proposition 4.4 of [Ra1], one needs to know exactly which weights can occur in calibrated representations. The reason that this naturally depends on a rank two classification is outlined in (d) below.

(b) The examples here illustrate (and clarify) results of [Ra1], [KL], [CG], [BM], [Ev], [Kr], [HO12 ]. Much of the power of the combinatorial methods which are now available is evident from the calculations in this paper, especially when one compares with the effort needed in other sources (for example [Xi], Chapt. 11).

(c) The explicit information here can be very useful for obtaining results on representations of $p$-adic groups (see, for example, [Lu3]).

(d) One hopes that eventually there will be a combinatorial construction of all irreducible representations of all affine Hecke algebras. I expect that such a construction will depend heavily on the rank two cases. This idea is analogous to the way that the rank two cases are the basic building blocks in the presentations of Coxeter groups by "braid" relations and the presentations of Kac-Moody Lie algebras (and quantum groups) by Serre relations.

The first section of this paper is a review of definitions and basic results about affine Hecke algebras and their representations. A few additional lemmas are proved in order to aid the proofs and constructions in later sections. The remainder of the sections detail the classification and construction of the irreducible representations of affine Hecke algebras of types $A_{1}, A_{1} \times A_{1}, A_{2}, C_{2}$ and $G_{2}$. In each case I have indicated how the results here relate to the "Langlands classification", the classification of Kazhdan and Lusztig [KL], and the results in [Ra1].

Acknowledgements. This paper is part of a series [Ra1-3] [RR1-2] on representations of affine Hecke algebras. During this work I have benefited from conversations with many people. To choose

\footnotetext{
* Research supported in part by National Science Foundation grant DMS-9622985, and a Postdoctoral Fellowship at Mathematical Sciences Research Institute.
} 
only a few, there were discussions with S. Fomin, F. Knop, L. Solomon, M. Vazirani and N. Wallach which played an important role in my progress. There were several times when I tapped into J. Stembridge's fountain of useful knowledge about root systems. D.-N. Verma helped at a crucial juncture by suggesting that I look at the paper of Steinberg. G. Benkart was a very patient listener on many occasions. H. Barcelo, P. Deligne, T. Halverson, R. Macpherson and R. Simion all gave large amounts of time to let me tell them my story and every one of these sessions was helpful to me in solidifying my understanding.

I single out Jacqui Ramagge with special thanks for everything she has done to help with this project: from the most mundane typing and picture drawing to deep intense mathematical conversations which helped to sort out many pieces of this theory. Her immense contribution is evident in that some of the papers in this series on representations of affine Hecke algebras are joint papers.

A portion of this research was done during a semester long stay at Mathematical Sciences Research Institute where I was supported by a Postdoctoral Fellowship. I thank MSRI and National Science Foundation for support of my research.

\section{Definitions AND PRELIMINARY RESUlts}

The Weyl group. Let $R$ be a reduced irreducible root system in $\mathbb{R}^{n}$, fix a set of positive roots $R^{+}$and let $\left\{\alpha_{1}, \ldots, \alpha_{n}\right\}$ be the corresponding simple roots in $R$. Let $W$ be the Weyl group corresponding to $R$. Let $s_{i}$ denote the simple reflection in $W$ corresponding to the simple root $\alpha_{i}$ and recall that $W$ can be presented by generators $s_{1}, s_{2}, \ldots, s_{n}$ and relations

$$
\begin{array}{clrl}
s_{i}^{2} & = & 1, & \text { for } 1 \leq i \leq n, \\
\underbrace{s_{i} s_{j} s_{i} \cdots}= & \underbrace{s_{j} s_{i} s_{j} \cdots}, & \text { for } i \neq j,
\end{array}
$$

where $m_{i j}=\left\langle\alpha_{i}, \alpha_{j}^{\vee}\right\rangle\left\langle\alpha_{j}, \alpha_{i}^{\vee}\right\rangle$, and $\alpha_{i}^{\vee}=2 \alpha_{i} /\left\langle\alpha_{i}, \alpha_{i}\right\rangle$.

The Iwahori-Hecke algebra. Fix $q \in \mathbb{C}^{*}$ such that $q$ is not a root of unity. The Iwahori-Hecke algebra $H$ is the associative algebra over $\mathbb{C}$ defined by generators $T_{1}, T_{2}, \ldots, T_{n}$ and relations

$$
\begin{array}{clr}
T_{i}^{2} & =\left(q-q^{-1}\right) T_{i}+1, & \text { for } 1 \leq i \leq n, \\
\underbrace{T_{i} T_{j} T_{i} \cdots}_{m_{i j} \text { factors }} & =\underbrace{T_{j} T_{i} T_{j} \cdots}_{m_{i j} \text { factors }}, & \text { for } i \neq j,
\end{array}
$$

where $m_{i j}$ are the same as in the presentation of $W$. For $w \in W$ define $T_{w}=T_{i_{1}} \cdots T_{i_{p}}$ where $s_{i_{1}} \cdots s_{i_{p}}=w$ is a reduced expression for $w$. By [Bou, Ch. IV $\S 2$ Ex. 23], the element $T_{w}$ does not depend on the choice of the reduced expression. The algebra $H$ has dimension $|W|$ and the set $\left\{T_{w}\right\}_{w \in W}$ is a basis of $H$.

The group $X$. The fundamental weights are the elements $\omega_{1}, \ldots, \omega_{n}$ of $\mathbb{R}^{n}$ given by $\left\langle\omega_{i}, \alpha_{j}^{\vee}\right\rangle=$ $\delta_{i j}$. The weight lattice is the $W$-invariant lattice in $\mathbb{R}^{n}$ given by

$$
P=\sum_{i=1}^{n} \mathbb{Z} \omega_{i} .
$$

Let $X$ be the abelian group $P$ except written multiplicatively. In other words,

$$
X=\left\{X^{\lambda} \mid \lambda \in P\right\}, \quad \text { and } \quad X^{\lambda} X^{\mu}=X^{\lambda+\mu}=X^{\mu} X^{\lambda}, \quad \text { for } \lambda, \mu \in P .
$$


Let $\mathbb{C}[X]$ denote the group algebra of $X$. There is a $W$-action on $X$ given by $w X^{\lambda}=X^{w \lambda}$ for $w \in W, X^{\lambda} \in X$, which we extend linearly to a $W$-action on $\mathbb{C}[X]$.

The affine Hecke algebra. The affine Hecke algebra $\tilde{H}$ associated to $R$ and $P$ is the algebra given by

$$
\tilde{H}=\mathbb{C}-\operatorname{span}\left\{T_{w} X^{\lambda} \mid w \in W, X^{\lambda} \in X\right\}
$$

where the multiplication of the $T_{w}$ is as in the Iwahori-Hecke algebra $H$, the multiplication of the $X^{\lambda}$ is as in $\mathbb{C}[X]$ and we impose the relation

$$
X^{\lambda} T_{i}=T_{i} X^{s_{i} \lambda}+\left(q-q^{-1}\right) \frac{X^{\lambda}-X^{s_{i} \lambda}}{1-X^{-\alpha_{i}}}, \quad \text { for } 1 \leq i \leq n \text { and } X^{\lambda} \in X
$$

This formulation of the definition of $\tilde{H}$ is due to Lusztig [Lu2] following work of Bernstein and Zelevinsky. The elements $T_{w} X^{\lambda}, w \in W, X^{\lambda} \in X$, form a basis of $\tilde{H}$.

Weights. Let

$$
T=\left\{\text { group homomorphisms } t: X \rightarrow \mathbb{C}^{*}\right\}
$$

The torus $T$ is an abelian group with a $W$-action given by $(w t)\left(X^{\lambda}\right)=t\left(X^{w^{-1} \lambda}\right)$. For any element $t \in T$ define the polar decomposition

$$
t=t_{r} t_{c}, \quad t_{r}, t_{c} \in T \text { such that } t_{r}\left(X^{\lambda}\right) \in \mathbb{R}_{>0}, \text { and }\left|t_{c}\left(X^{\lambda}\right)\right|=1
$$

for all $X^{\lambda} \in X$. Let $Q^{\vee}=\sum_{i} \mathbb{Z} \alpha_{i}^{\vee}$. There is a unique $\mu \in \mathbb{R}^{n}$ and a unique $\nu \in \mathbb{R}^{n} / Q^{\vee}$ such that

$$
t_{r}\left(X^{\lambda}\right)=e^{\langle\mu, \lambda\rangle} \quad \text { and } \quad t_{c}\left(X^{\lambda}\right)=e^{2 \pi i\langle\nu, \lambda\rangle}, \quad \text { for all } \lambda \in P .
$$

In this way we identify the sets $T_{r}=\left\{t \in T \mid t=t_{r}\right\}$ and $T_{c}=\left\{t \in T \mid t=t_{c}\right\}$ with $\mathbb{R}^{n}$ and $\mathbb{R}^{n} / Q^{\vee}$, respectively.

\section{Central characters.}

Theorem 1.4. (Bernstein, Zelevinsky, Lusztig $\left[\right.$ Lu1, 8.1]) The center of $\tilde{H}$ is $\mathbb{C}[X]^{W}=\{f \in$ $\mathbb{C}[X] \mid w f=f\}$.

Since $\tilde{H}$ has countable dimension, Dixmier's version of Schur's lemma implies that $Z(\tilde{H})$ acts on an irreducible $\tilde{H}$-module $M$ by scalars. Let $t \in T$ be such that

$$
p M=t(p) M, \quad \text { for all } p \in Z(\tilde{H})
$$

Since $Z(\tilde{H})=\mathbb{C}[X(T)]^{W}$ it follows that $t(p(X))=(w t)(p(X))$ for all $w \in W$. The $W$-orbit $W t$ of $t$ is the central character of $M$. We shall often abuse notation and refer to any weight $s \in W t$ as "the central character" of $M$.

Weight spaces. Let $M$ be a finite dimensional $\tilde{H}$-module. For each $t \in T$ the $t$-weight space of $M$ and the generalized t-weight space are the subspaces

$$
\begin{aligned}
M_{t} & =\left\{m \in M \mid X^{\lambda} m=t\left(X^{\lambda}\right) m \text { for all } X^{\lambda} \in X\right\} \quad \text { and } \\
M_{t}^{\text {gen }} & =\left\{m \in M \mid \text { for each } X^{\lambda} \in X,\left(X^{\lambda}-t\left(X^{\lambda}\right)\right)^{k} m=0 \text { for some } k \in \mathbb{Z}_{>0}\right\},
\end{aligned}
$$


respectively. If $M_{t}^{\text {gen }} \neq 0$ then $M_{t} \neq 0$. In general $M \neq \bigoplus_{t \in T} M_{t}$, but we do have

$$
M=\bigoplus_{t \in T} M_{t}^{\text {gen }}
$$

This is a decomposition of $M$ into Jordan blocks for the action of $\mathbb{C}[X]$. The set of weights of $M$ is the set

$$
\operatorname{supp}(M)=\left\{t \in T \mid M_{t}^{\text {gen }} \neq 0\right\}
$$

The calibration graph. Let $t \in T$. Define a graph $\Gamma(t)$ with

$$
\begin{aligned}
\text { Vertices: } & W t, \\
\text { Edges: } & w t \longleftrightarrow s_{i} w t, \quad \text { if } \quad(w t)\left(X^{\alpha_{i}}\right) \neq q^{ \pm 2} .
\end{aligned}
$$

Proposition 1.6. ([Ra1] Proposition 2.12) Let $M$ be a finite dimensional irreducible $\tilde{H}$-module with central character $t$. Then

$$
\operatorname{dim}\left(M_{s}^{\text {gen }}\right)=\operatorname{dim}\left(M_{s^{\prime}}^{\text {gen }}\right)
$$

if $s$ and $s^{\prime}$ are in the same connected component of the calibration graph $\Gamma(t)$.

If $t \in T$ define

$$
P(t)=\left\{\alpha>0 \mid t\left(X^{\alpha}\right)=q^{ \pm 2}\right\} \quad \text { and } \quad Z(t)=\left\{\alpha>0 \mid t\left(X^{\alpha}\right)=1\right\} .
$$

For each subset $J \subseteq P(t)$ define

$$
\mathcal{F}^{(t, J)}=\{w \in W \mid R(w) \cap Z(t)=\emptyset, \quad R(w) \cap P(t)=J\},
$$

where $R(w)=\{\alpha>0 \mid w \alpha<0\}$ is the inversion set of $w$. Define a placed shape to be a pair $(t, J)$ such that $t \in T, J \subseteq P(t)$ and $\mathcal{F}^{(t, J)} \neq \emptyset$. The elements of the set $\mathcal{F}^{(t, J)}$ are called standard tableaux of shape $(t, J)$.

Proposition 1.9. ([Ra1] Theorem 2.14) Let $t \in T$. The connected components of the calibration graph $\Gamma(t)$ are the sets

$$
\left\{w t \mid w \in \mathcal{F}^{(t, J)}\right\}, \quad J \subseteq P(t), \quad \text { such that } \mathcal{F}^{(t, J)} \neq \emptyset
$$

Calibrated representations. A finite dimensional $\tilde{H}$-module $M$ is calibrated if $M_{t}^{\text {gen }}=M_{t}$, for all $t \in T$.

Proposition 1.10. ([Ra1] Proposition 4.2)

(a) An irreducible $\tilde{H}$-module $M$ is calibrated if and only if $\operatorname{dim}\left(M_{t}^{\text {gen }}\right)=1$ for all weights $t$ of $M$.

(b) If $M$ is an irreducible $\tilde{H}$-module with regular central character $t$ (i.e. $Z(t)=\emptyset$ ) then $M$ is calibrated.

Let $\alpha_{i}$ and $\alpha_{j}$ be simple roots in $R$ and let $R_{i j}$ be the rank two root subsystem of $R$ which is generated by $\alpha_{i}$ and $\alpha_{j}$. Let $W_{i j}$ be the Weyl group of $R_{i j}$, the subgroup of $W$ generated by 
the simple reflections $s_{i}$ and $s_{j}$. A weight $t \in T$ is calibratable for $R_{i j}$ if one of the following two conditions holds:

(a) $t\left(X^{\alpha}\right) \neq 1$ for all $\alpha \in R_{i j}$

(b) $R_{i j}$ is of type $C_{2}$ or $G_{2}$ (assume that $\alpha_{i}$ is the long root and $\alpha_{j}$ is the short root), $u t\left(X^{\alpha_{i}}\right)=q^{2}$ and $u t\left(X^{\alpha_{j}}\right)=1$ for some $u \in W_{i j}$, and $t\left(X^{\alpha_{i}}\right) \neq 1$ and $t\left(X^{\alpha_{j}}\right) \neq 1$.

A placed skew shape is a placed shape $(t, J)$ such that for all $w \in \mathcal{F}^{(t, J)}$ and all pairs $\alpha_{i}, \alpha_{j}$ of simple roots in $R$ the weight $w t$ is calibratable for $R_{i j}$.

Theorem 1.11. ([Ra1] Theorem 3.1 and Proposition 4.1)

(a) Let $(t, J)$ be a placed skew shape and let $\mathcal{F}^{(t, J)}$ be the set of standard tableaux of shape $(t, J)$. Define

$$
\tilde{H}^{(t, J)}=\mathbb{C}-\operatorname{span}\left\{v_{w} \mid w \in \mathcal{F}^{(t, J)}\right\},
$$

so that the symbols $v_{w}$ are a labeled basis of the vector space $\tilde{H}^{(t, J)}$. Then the following formulas make $\tilde{H}^{(t, J)}$ into an irreducible $\tilde{H}$-module: For each $w \in \mathcal{F}^{(t, J)}$,

$$
\begin{array}{ll}
X^{\lambda} v_{w}=(w t)\left(X^{\lambda}\right) v_{w}, & \text { for } X^{\lambda} \in X, \text { and } \\
T_{i} v_{w}=\left(T_{i}\right)_{w w} v_{w}+\left(q^{-1}+\left(T_{i}\right)_{w w}\right) v_{s_{i} w}, & \text { for } 1 \leq i \leq n,
\end{array}
$$

where $\left(T_{i}\right)_{w w}=\frac{q-q^{-1}}{1-(w t)\left(X^{-\alpha_{i}}\right)}$, and we set $v_{s_{i} w}=0$ if $s_{i} w \notin \mathcal{F}^{(t, J)}$.

(b) If $M$ is an irreducible calibrated representation such that $\operatorname{supp}(M)=\left\{w t \mid w \in \mathcal{F}^{(t, J)}\right\}$ for some placed skew shape $(t, J)$ then $M$ is isomorphic to the module $\tilde{H}^{(t, J)}$ constructed in (a).

Remark 1.12. It follows from the results of Rodier [Ro] that if $M$ is an irreducible $\tilde{H}$-module with regular central character (i.e. $Z(t)=\emptyset$ ) then $M$ satisfies the hypothesis of the statement of Theorem 1.11 (b).

Langlands classification. The following discussion follows the work of Evens [Ev] and [KL, $\S 8]$. For this subsection it is convenient to assume that $q \in \mathbb{R}_{>0}$ and $q \neq 1$. For the general case see $[\mathrm{KL}, \S 8]$. Let $t \in T$ and let $t=t_{r} t_{c}$ be the polar decomposition of $t$. Define

$$
\nu(t) \in \sum_{i=1}^{n} \mathbb{R} \alpha_{i}^{\vee} \quad \text { by requiring } \quad t_{r}\left(X^{\lambda}\right)=q^{2\langle\lambda, \nu(t)\rangle}, \quad \text { for all } \lambda \in P .
$$

A finite dimensional $\tilde{H}$-module $M$ is tempered if for all weights $t$ of $M$ (as defined in (1.5)) we have

$$
\left\langle\omega_{i}, \nu(t)\right\rangle \leq 0, \quad \text { for all } 1 \leq i \leq n
$$

The module $M$ is square integrable if $\left\langle\omega_{i}, \nu(t)\right\rangle<0$ for all $1 \leq i \leq n$ and all weights $t$ of $M$.

Let $I$ be a subset of the simple roots and let $\tilde{H}_{I}$ be the subalgebra of $\tilde{H}$ generated by $T_{i}, i \in I$, and all $X^{\lambda} \in X$. We shall say that a finite dimensional $\tilde{H}_{I}$-module is tempered if $I$ is the maximal set such that for all weights $t$ of $M$,

$$
\left\langle\omega_{i}, \nu(t)\right\rangle \leq 0, \quad \text { for all } i \in I
$$


Theorem 1.13. (see $\left.\left[E_{V}\right]\right)$ Let $I \subseteq\{1,2 \ldots, n\}$ and let $\mathcal{T}$ be an irreducible tempered representation of $\tilde{H}_{I}$.

(a) $M_{\mathcal{T}, I}=\operatorname{Ind}_{\tilde{H}_{I}}^{\tilde{H}}(\mathcal{T})$ has a unique irreducible quotient $L_{\mathcal{T}, I}$.

(b) Every irreducible $\tilde{H}$-module is isomorphic to $L_{\mathcal{T}, I}$ for some pair $(\mathcal{T}, I)$.

(c) If $L_{\mathcal{T}, I} \cong L_{\mathcal{T}^{\prime}, I^{\prime}}$ then $I=I^{\prime}$ and $\mathcal{T} \cong \mathcal{T}^{\prime}$ as $\tilde{H}_{I^{-}}$-modules.

The Langlands parameters of an irreducible $\tilde{H}$-module $M$ are given by the pair $(\mathcal{T}, I)$ specified by Theorem 1.13 (b).

Classification by indexing triples. Kazhdan and Lusztig [KL] (see also the important work of Ginzburg [CG]) gave a refinement of the Langlands classification. Let $G$ be the simple complex algebraic group with root system $R$ and weight lattice $P$. An indexing triple $(s, u, \rho)$ consists of

$$
\begin{gathered}
\text { a semisimple element } s \in G, \quad \text { such that } \quad s u s^{-1}=u^{q^{2}}, \\
\text { a unipotent element } u \in G,
\end{gathered}
$$

and an irreducible representation $\rho$ of the component group $A(s, u)=Z_{G}(s, u) / Z_{G}(s, u)^{\circ}$, where $Z_{G}(s, u)=Z_{G}(s) \cap Z_{G}(u)$. Let $K\left(\mathcal{B}_{s, u}\right)$ be the $K$-theory of the variety

$$
\mathcal{B}_{s, u}=\{\text { Borel subgroups of } G \text { containing both } s \text { and } u\} \text {. }
$$

By a theorem of Lusztig [Lu4] $K\left(\mathcal{B}_{s, u}\right)$ is an $\tilde{H}$-module. The group $A(s, u)$ also acts on $K\left(\mathcal{B}_{s, u}\right)$ and this action commutes with the action of $\tilde{H}$. The standard modules $M_{s, u, \rho}$ are the $\tilde{H}$-modules given by the decomposition

$$
K\left(\mathcal{B}_{s, u}\right)=\bigoplus_{\rho} M_{s, u, \rho} \otimes \rho,
$$

where the sum is over all irreducible representations of $A(s, u)$.

Theorem 1.14. $[K L]$

(a) If $M_{s, u, \rho} \neq 0$ then it has a unique simple quotient $L_{s, u, \rho}$.

(b) Every simple $\tilde{H}$-module isomorphic to some $L_{s, u, \rho}$.

(c) If $L_{s, u, \rho} \cong L_{s^{\prime}, u^{\prime}, \rho^{\prime}}$ then there is a $g \in G$ such that $s^{\prime}=g s g^{-1}, u^{\prime}=g u g^{-1}$, and $\rho^{\prime}=\rho$.

In this way each irreducible $\tilde{H}$-module corresponds to a unique (up to conjugation) indexing triple. One can replace $u$ by $n=\ln u$ in the Lie algebra $\mathfrak{g}=\operatorname{Lie}(G)$ (see [CG, Ch. 8]) so that an indexing triple is

$$
\begin{aligned}
& \text { a semisimple element } s \in G, \quad \text { such that } \quad \operatorname{Ad}(s) n=q^{2} n, \\
& \text { a nilpotent element } n \in \mathfrak{g},
\end{aligned}
$$

and an irreducible representation $\rho$ of the component group $A(s, n)=Z_{G}(s, n) / Z_{G}(s, n)^{\circ}$, where $Z_{G}(s, n)=Z_{G}(s) \cap Z_{G}(n)$ and $Z_{G}(n)$ is taken with respect to the adjoint action of $G$ on $\mathfrak{g}$. We will use this form of the indexing triples in the examples in later sections.

Principal series modules. Let $t \in T$ and let $\mathbb{C} v_{t}$ be the one dimensional $\mathbb{C}[X]$-module corresponding to the character $t: X \rightarrow \mathbb{C}^{*}$. Specifically, $\mathbb{C} v_{t}$ is the one dimensional vector space with basis $\left\{v_{t}\right\}$ and $\mathbb{C}[X]$-action given by

$$
X^{\lambda} v_{t}=t\left(X^{\lambda}\right) v_{t}, \quad \text { for all } X^{\lambda} \in X .
$$


The principal series module corresponding to $t$ is the $\tilde{H}$-module

$$
M(t)=\operatorname{Ind}_{\mathbb{C} X}^{\tilde{H}}\left(\mathbb{C} v_{t}\right) .
$$

Theorem 1.15. [Ma]

(a) Every irreducible $\tilde{H}$-module $M$ with central character $t$ is a composition factor of the principal series module $M(t)$.

(b) If $w \in W$ and $t \in T$ then $M(t)$ and $M(w t)$ have the same composition factors.

Theorem 1.16. (Kato's irreducibility criterion [Ka]) Let $t \in T$ and let $P(t)=\left\{\alpha>0 \mid t\left(X^{\alpha}\right)=\right.$ $\left.q^{ \pm 2}\right\}$. The principal series module $M(t)$ is irreducible if and only if $P(t)=\emptyset$.

Remark. Kato actually proves a more general result and thus needs a further condition for irreducibility. We have simplified matters by specifying the weight lattice $P$ in our construction of the affine Hecke algebra. One can use any $W$-invariant lattice in $\mathbb{R}^{n}$ and Kato works in this more general situation. When the one uses the weight lattice $P$, a result of Steinberg [St, 4.2, 5.3] says that the stabilizer $W_{t}$ of a point $t \in T$ under the action of $W$ is always a reflection group. Because of this Kato's criterion takes a simpler form.

Weights of induced modules. If $I \subseteq\{1, \ldots, n\}$ define $\tilde{H}_{I}$ to be the subalgebra of $\tilde{H}$ generated by $T_{i}, i \in I$, and all $X^{\lambda} \in X$.

Lemma 1.17. Let $t \in T$ such that $t\left(X^{\alpha_{i}}\right)=q^{2}$ for all $i \in I$ and let $\mathbb{C} v_{t}$ be the one dimensional $\tilde{H}_{I}$-module with basis $\left\{v_{t}\right\}$ and $\tilde{H}_{I}$-action given by

$$
T_{i} v_{t}=q v_{t}, \quad \text { for } i \in I, \quad \text { and } \quad X^{\lambda} v_{t}=t\left(X^{\lambda}\right) v_{t}, \quad \text { for all } X^{\lambda} \in X
$$

Let $W / W_{I}$ be the set of minimal length coset representatives of cosets of $W_{I}$ in $W$. Then the weights of the $\tilde{H}$-module $M=\operatorname{Ind}_{\tilde{H}_{I}}^{\tilde{\tilde{C}}}\left(\mathbb{C} v_{t}\right)$ are $w t, w \in W / W_{I}$, and

$$
\operatorname{dim}\left(M_{w t}^{\mathrm{gen}}\right)=\left(\# \text { of } u \in W / W_{I} \text { such that } u t=w t\right) .
$$

Proof. The module $M$ has basis $\left\{T_{w} \otimes v_{t} \mid w \in W / W_{I}\right\}$. By writing $T_{w}=T_{i_{1}} \cdots T_{i_{p}}$ for a reduced word $w=s_{i_{1}} \ldots s_{i_{p}}$ and inductively using the defining relation (1.2) we get

$$
\begin{aligned}
X^{\lambda}\left(T_{w} \otimes v_{t}\right) & =t\left(X^{w^{-1} \lambda}\right)\left(T_{w} \otimes v_{t}\right)+\sum_{v<w} a_{v}(t)\left(T_{v} \otimes v_{t}\right) \\
& =(w t)\left(X^{\lambda}\right)\left(T_{w} \otimes v_{t}\right)+\sum_{v<w} a_{v}(t)\left(T_{v} \otimes v_{t}\right),
\end{aligned}
$$

where the sum is over $v \in W$ which are less than $w$ in Bruhat order and $a_{v}(t) \in \mathbb{C}$. This shows that the eigenvalues of $X^{\lambda}$ on $M$ are $(w t)\left(X^{\lambda}\right)$. The result follows by counting the multiplicity of each eigenvalue.

The $\tau$ operators. The maps $\tau_{i}: M_{t}^{\text {gen }} \rightarrow M_{s_{i} t}^{\text {gen }}$ defined below are local operators on $M$ in the sense that they act on each weight space $M_{t}^{\text {gen }}$ of $M$ separately. The operator $\tau_{i}$ is only defined on weight spaces $M_{t}^{\text {gen }}$ such that $t\left(X^{\alpha_{i}}\right) \neq 1$. 
Proposition 1.18. ([Ra1] Proposition 2.7) Let $t \in T$ such that $t\left(X^{\alpha_{i}}\right) \neq 1$ and let $M$ be a finite dimensional $\tilde{H}$-module. Define

$$
\begin{array}{rlc}
\tau_{i}: M_{t}^{\text {gen }} & \longrightarrow & M_{s_{i} t}^{\text {gen }} \\
m & \longmapsto & \left(T_{i}-\frac{q-q^{-1}}{1-X^{-\alpha_{i}}}\right) m .
\end{array}
$$

(a) The map $\tau_{i}$ : $M_{t}^{\text {gen }} \longrightarrow M_{s_{i} t}^{\text {gen }}$ is well defined.

(b) As operators on $M_{t}^{\text {gen }}, \quad X^{\lambda} \tau_{i}=\tau_{i} X^{s_{i} \lambda}$, for all $X^{\lambda} \in X$.

(c) As operators on $M_{t}^{\text {gen }}, \quad \tau_{i} \tau_{i}=\frac{\left(q-q^{-1} X^{\alpha_{i}}\right)\left(q-q^{-1} X^{-\alpha_{i}}\right)}{\left(1-X^{\alpha_{i}}\right)\left(1-X^{-\alpha_{i}}\right)}$.

(d) Let $1 \leq i \neq j \leq n$ and let $m_{i j}$ be as in (1.1). Then $\underbrace{\tau_{i} \tau_{j} \tau_{i} \cdots}_{m_{i j} \text { factors }}=\underbrace{\tau_{j} \tau_{i} \tau_{i} \cdots}_{m_{i j} \text { factors }}$

whenever both sides are well defined operators on $M_{t}^{\text {gen }}$.

Lemma 1.19. Let $t \in T$ such that $t\left(X^{\alpha_{i}}\right)=1$ and suppose that $M$ is an $\tilde{H}$-module such that $M_{t}^{\text {gen }} \neq 0$. Let $W_{t}$ be the stabilizer of $t$ under the action of $W$ on $T$. Assume that $\bar{w} \in W / W_{t}$ is such that $t$ and $\bar{w} t$ are in the same connected component of $\Gamma(t)$. Let $w$ be a minimal length coset representative of $\bar{w}$. Then

(a) $\operatorname{dim}\left(M_{w t}^{\text {gen }}\right) \geq 2$, and

(b) If $M_{s_{j} w t}^{\text {gen }}=0$ then $(\bar{w} t)\left(X^{\alpha_{j}}\right)=q^{ \pm 2}$ and $\left\langle w^{-1} \alpha_{j}, \alpha_{i}^{\vee}\right\rangle=0$.

Proof. Let $M(t)$ be the two dimensional principal series module for the affine Hecke algebra $\tilde{H} A_{1}$ of type $A_{1}$ (see $\S 2$ central character $t_{o}$ ). Then $M(t)=M(t)_{t}^{\text {gen }}$ and has basis $\left\{v_{t}, T_{1} v_{t}\right\}$. Let $n_{t}$ be a nonzero weight vector in $M_{t}$. There is a unique $\tilde{H} A_{1}$-module homomorphism

$$
\begin{gathered}
M(t) \quad \longrightarrow M \\
v_{t} \longmapsto n_{t}
\end{gathered}
$$

where we view $M$ as an $\tilde{H} A_{1}$-module by restriction to the parabolic subalgebra $\tilde{H}_{\{i\}} \subseteq \tilde{H}$. This homomorphism must be an injection since $M(t)$ is irreducible. Thus the vectors $n_{t}, T_{i} n_{t}$ span a two dimensional subspace of $M_{t}^{\text {gen }}$ and $X^{\lambda} \in X$ acts on this subspace by the matrix

$$
\phi_{t}\left(X^{\lambda}\right)=t\left(X^{\lambda}\right)\left(\begin{array}{cc}
1 & \left(q-q^{-1}\right)\left\langle\lambda, \alpha_{i}^{\vee}\right\rangle \\
0 & 1
\end{array}\right)
$$

Let $w=s_{i_{1}} \cdots s_{i_{p}}$ be a reduced expression of $w$. Since $t$ and $w t$ are in the same connected component of $\Gamma(t)$ we can use Proposition 1.18 (c) to show that the map

$$
\tau_{w}=\tau_{i_{1}} \cdots \tau_{i_{p}}: M_{t}^{\text {gen }} \longrightarrow M_{w t}^{\text {gen }}
$$

is well defined and bijective. Thus the vectors $\tau_{w} n_{t}, \tau_{w} T_{i} n_{t}$ span a two dimensional subspace of $M_{w t}^{\text {gen }}$ and, by Proposition 1.18 (b) $X^{\lambda} \in X$ acts on this subspace by the matrix

$$
\phi_{w t}\left(X^{\lambda}\right)=t\left(X^{w^{-1} \lambda}\right)\left(\begin{array}{cc}
1 & \left(q-q^{-1}\right)\left\langle w^{-1} \lambda, \alpha_{i}^{\vee}\right\rangle \\
0 & 1
\end{array}\right)
$$


This proves (a). Then

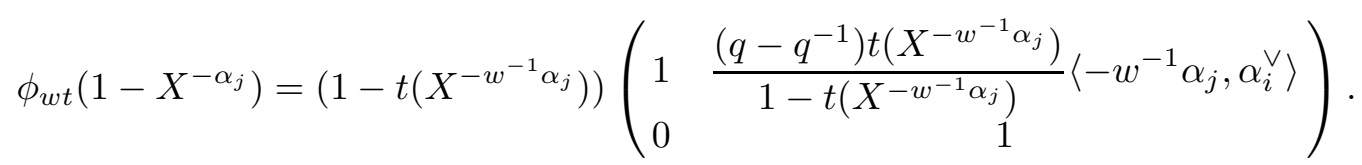

Since $M_{s_{j} w t}^{\text {gen }}=0, \tau_{j}: M_{w t}^{\text {gen }} \rightarrow M_{s_{j} w t}^{\text {gen }}$ is the zero map and so

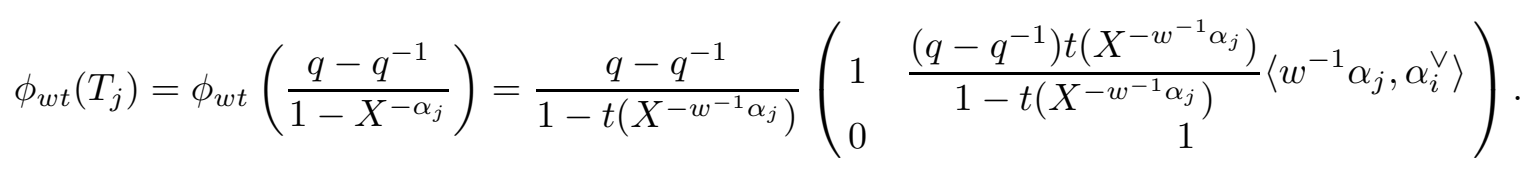

The relation $T_{j}^{2}=\left(q-q^{-1}\right) T_{j}+1$ is the same as $\left(T_{j}-q\right)\left(T_{j}+q^{-1}\right)=0$. This relation forces $\phi_{w t}\left(T_{j}\right)$ to have Jordan blocks of size 1 and eigenvalues $\pm q^{ \pm 1}$. It follows that $t\left(X^{w^{-1} \alpha_{j}}\right)=q^{ \pm 2}$ and $\left\langle w^{-1} \alpha_{j}, \alpha_{i}^{\vee}\right\rangle=0$.

\section{Classification for $A_{1}$}

The root system $R$ for $A_{1}$ has one simple root $\alpha_{1}$ and fundamental weight $\omega_{1}=\frac{1}{2} \alpha_{1}$.

Irreducible representations. Table 2.1 lists the irreducible $\tilde{H}$-modules by their central characters. The sets $P(t)$ and $Z(t)$ are as given in (1.7) and correspond to the choice of representative for the central character displayed in Figure 2.1. The Langlands parameters usually consist of a pair $(\mathcal{T}, I)$ where $I$ is a subset of $\{1\}$ and $\mathcal{T}$ is a tempered representation for the parabolic subalgebra $\tilde{H}_{I}$. In our cases the tempered representation $\mathcal{T}$ of $\tilde{H}_{I}$ is completely determined by a character $t \in T$. Specifically, $\mathcal{T}$ is the only tempered representation of $\tilde{H}_{I}$ which has $t$ as a weight. In the labeling in Table 2.1 we have replaced the representation $\mathcal{T}$ by the weight $t$. The nilpotent element $e_{\alpha_{1}}$ is a representative of the root space $\mathfrak{g}_{\alpha_{1}}$ for the Lie algebra $\mathfrak{g}=\mathfrak{s l}_{2}$. For each calibrated module with central character $t$ we have listed the subset $J \subseteq P(t)$ such that $(t, J)$ is the corresponding placed skew shape (see Theorem 1.11). The abbreviation 'nc' indicates modules that are not calibrated.

$\begin{array}{ccccccc}\begin{array}{c}\text { Central } \\ \text { character }\end{array} & P(t) & Z(t) & \text { Dimension } & \begin{array}{c}\text { Langlands } \\ \text { parameters }\end{array} & \begin{array}{c}\text { Indexing } \\ \text { triple }\end{array} & \begin{array}{c}\text { Calibration } \\ \text { set } J\end{array} \\ t_{a} & \left\{\alpha_{1}\right\} & \emptyset & 1 & \begin{array}{c}\left(t_{a}, \emptyset\right) \\ \text { tempered }\end{array} & \begin{array}{c}\left(t_{a}, 0,1\right) \\ \left(t_{a}, e_{\alpha_{1}}, 1\right)\end{array} & \begin{array}{c}\emptyset \\ \left.\alpha_{1}\right\}\end{array} \\ t_{b} & \emptyset & \emptyset & 2 & \left(t_{b}, \emptyset\right) & \left(t_{b}, 0,1\right) & \emptyset \\ t_{o} & \emptyset & \left\{\alpha_{1}\right\} & 2 & \text { tempered } & \left(t_{o}, 0,1\right) & \mathrm{nc}\end{array}$

Table 2.1. Irreducible representations

Figure 2.1 displays the real parts of the central characters in Table 2.1. If $t \in T$ then the polar decomposition $t=t_{r} t_{c}$ determines an element $\mu \in \mathbb{R}^{n}$ such that $t_{r}\left(X^{\lambda}\right)=e^{\langle\lambda, \mu\rangle}$ (see (1.3)). 
For each central character $t_{p}$ the point labeled by $p$ in Figure 2.1 is the graph of the corresponding $\mu_{p} \in \mathbb{R}^{n}$. Assume (for pictorial convenience) that $q$ is a positive real number and let

$$
H_{\alpha_{1}}=\left\{x \in \mathbb{R} \mid\left\langle\alpha_{1}, x\right\rangle=0\right\}, \quad \text { and } \quad H_{\alpha_{1} \pm \delta}=\left\{x \in \mathbb{R} \mid\left\langle\alpha_{1}, x\right\rangle=\ln \left(q^{ \pm 2}\right)\right\} \text {. }
$$

The | marks indicate the (affine) hyperplanes $H_{\alpha_{1} \pm \delta}$.

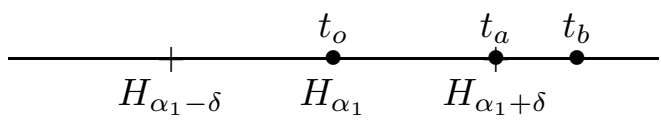

Figure 2.1. Real parts of central characters in Table 2.1

Tempered and square integrable representations. The tempered (resp. square integrable) $\tilde{H}$-modules are the ones which have all their weight spaces in the closure (resp. interior) of the dotted region of Figure 2.2.

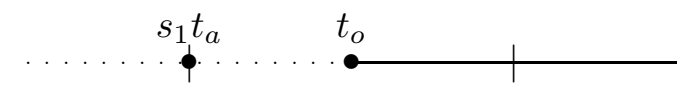

Figure 2.2. Real parts of weights of tempered representations

The irreducible tempered representations with real central character can be indexed by the irreducible representations of the symmetric group $S_{2}$ (see [BM]). These representations are indexed by the partitions $(2),\left(1^{2}\right)$ of 2 . Let $e_{\alpha_{1}}$ be an element of the root space $\mathfrak{g}_{\alpha_{1}}$ for the Lie algebra $\mathfrak{g}=\mathfrak{s l}_{2}$. The two nilpotent orbits in $\mathfrak{g}$ and the corresponding tempered representations of $\tilde{H}$ are as in Table 2.2 .

Nilpotent orbit Indexing triple Square integrable $W$ representation

$\begin{array}{cccc}\text { regular } & \left(t_{a}, e_{\alpha_{1}}, 1\right) & \text { yes } & \left(1^{2}\right) \\ 0 & \left(t_{o}, 0,1\right) & \text { no } & (2)\end{array}$

Table 2.2. Tempered representations and the Springer correspondence
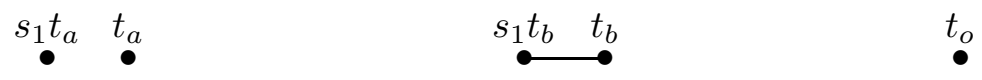

Figure 2.3. Calibration graphs for central characters in Table 2.1

\section{The analysis.}

Central character $t_{a}$ : There are two one-dimensional representations $\mathbb{C} v_{a}$ and $\mathbb{C} v_{s_{1} a}$ with central character $t_{a}$. These representations are given explicitly by

$$
\begin{aligned}
X^{\lambda} v_{a} & =t_{a}\left(X^{\lambda}\right) v_{a}, \\
T_{1} v_{a} & =q v_{a},
\end{aligned} \quad \text { and } \quad \begin{aligned}
X^{\lambda} v_{s_{1} a} & =\left(s_{1} t_{a}\right)\left(X^{\lambda}\right) v_{s_{1} a} \\
T_{1} v_{s_{1} a} & =-q^{-1} v_{s_{1} a}
\end{aligned}
$$

respectively. One uses Theorem 1.15 and the fact that the principal series module $M\left(t_{a}\right)$ is two dimensional to conclude that $\mathbb{C} v_{a}$ and $\mathbb{C} v_{s_{1} a}$ are the only irreducible representations of $\tilde{H}$ with central character $t_{a}$. 
Central character $t_{b}$ : By Theorem 1.15 and Kato's irreducibility criterion, Theorem 1.16, the only irreducible representation with central character $t_{b}$ is the principal series module $M\left(t_{b}\right)$. Alternatively, this module can be constructed by applying Theorem 1.11 to the placed skew shape $\left(t_{b}, \emptyset\right)$.

Central character $t_{o}$ : The weights given by $t_{o}\left(X^{\omega_{1}}\right)= \pm 1$ are the two central characters $t_{o} \in T$ which satisfy $P(t)=\emptyset, Z(t)=\left\{\alpha_{1}\right\}$. In either case Kato's irreducibility criterion (Theorem 1.16) tells us that the principal series module $M\left(t_{o}\right)$ is irreducible. This module has basis $\left\{v_{t}, T_{1} v_{t}\right\}$ and action given by

$$
\phi\left(X^{\lambda}\right)=t\left(X^{\lambda}\right)\left(\begin{array}{cc}
1 & \left(q-q^{-1}\right)\left\langle\lambda, \alpha_{1}^{\vee}\right\rangle \\
0 & 1
\end{array}\right) \quad \text { and } \quad \phi\left(T_{1}\right)=\left(\begin{array}{cc}
0 & 1 \\
1 & q-q^{-1}
\end{array}\right) .
$$

\section{Classification for $A_{1} \times A_{1}$}

The affine Hecke algebra of $A_{1} \times A_{1}$ is naturally isomorphic to $\tilde{H} A_{1} \otimes \tilde{H} A_{1}$. The finite dimensional irreducible representations of $\tilde{H} A_{1} \otimes \tilde{H} A_{1}$ are all of the form $M \otimes N$ where $M$ and $N$ are finite dimensional irreducible representations of $\tilde{H} A_{1}$. 


\section{Classification for $A_{2}$}

The root system $R$ for $A_{2}$ has simple roots $\alpha_{1}$ and $\alpha_{2}$, fundamental weights $\omega_{1}$ and $\omega_{2}$, and

$$
\begin{array}{ll}
\left\langle\alpha_{1}, \alpha_{2}^{\vee}\right\rangle=-1 & \omega_{1}=\frac{1}{3}\left(2 \alpha_{1}+\alpha_{2}\right) \\
\left\langle\alpha_{2}, \alpha_{1}^{\vee}\right\rangle=-1, & \omega_{2}=\frac{1}{3}\left(\alpha_{1}+2 \alpha_{2}\right),
\end{array} \quad \text { and } \quad \begin{aligned}
& \alpha_{1}=2 \omega_{1}-\omega_{2} \\
& \alpha_{2}=-\omega_{1}+2 \omega_{2} .
\end{aligned}
$$

Irreducible representations. Table 4.1 lists the irreducible $\tilde{H}$-modules by their central characters. The sets $P(t)$ and $Z(t)$ are as given in (1.7) and correspond to the choice of representative for the central character displayed in Figure 4.1. The Langlands parameters usually consist of a pair $(\mathcal{T}, I)$ where $I$ is a subset of $\{1,2\}$ and $\mathcal{T}$ is a tempered representation for the parabolic subalgebra $\tilde{H}_{I}$. In our cases the tempered representation $\mathcal{T}$ of $\tilde{H}_{I}$ is completely determined by a character $t \in T$. Specifically, $\mathcal{T}$ is the only tempered representation of $\tilde{H}_{I}$ which has $t$ as a weight. In the labeling in Table 4.1 we have replaced the representation $\mathcal{T}$ by the weight $t$. The nilpotent elements $e_{\alpha_{1}}$ and $e_{\alpha_{2}}$ are representatives of the root spaces $\mathfrak{g}_{\alpha_{1}}$ and $\mathfrak{g}_{\alpha_{2}}$, respectively, where $\mathfrak{g}$ is the Lie algebra $\mathfrak{g}=\mathfrak{s l}_{3}$. For each calibrated module with central character $t$ we have listed the subset $J \subseteq P(t)$ such that $(t, J)$ is the corresponding placed skew shape (see Theorem 1.11). The abbreviation 'nc' indicates modules that are not calibrated.

\begin{tabular}{|c|c|c|c|c|c|c|}
\hline $\begin{array}{l}\text { Central } \\
\text { character }\end{array}$ & $P(t)$ & $Z(t)$ & Dimension & $\begin{array}{l}\text { Langlands } \\
\text { parameters }\end{array}$ & $\begin{array}{l}\text { Indexing } \\
\text { triple }\end{array}$ & $\begin{array}{c}\text { Calibration } \\
\text { set } J\end{array}$ \\
\hline$t_{a}$ & $\left\{\alpha_{1}, \alpha_{2}\right\}$ & $\emptyset$ & $\begin{array}{l}1 \\
2 \\
2 \\
1\end{array}$ & $\begin{array}{c}\left(t_{a}, \emptyset\right) \\
\left(s_{1} t_{a},\{2\}\right) \\
\left(s_{2} t_{a},\{1\}\right) \\
\text { tempered }\end{array}$ & $\begin{array}{c}\left(t_{a}, 0,1\right) \\
\left(t_{a}, e_{\alpha_{2}}, 1\right) \\
\left(t_{a}, e_{\alpha_{1}}, 1\right) \\
\left(t_{a}, e_{\alpha_{1}}+e_{\alpha_{2}}, 1\right)\end{array}$ & $\begin{array}{c}\emptyset \\
\left\{\alpha_{2}\right\} \\
\left\{\alpha_{1}\right\} \\
\left\{\alpha_{1}, \alpha_{2}\right\}\end{array}$ \\
\hline$t_{b}$ & $\left\{\alpha_{2}\right\}$ & $\emptyset$ & $\begin{array}{l}3 \\
3\end{array}$ & $\begin{array}{c}\left(t_{b}, \emptyset\right) \\
\left(s_{2} t_{b},\{2\}\right)^{\dagger}\end{array}$ & $\begin{array}{c}\left(t_{b}, 0,1\right) \\
\left(t_{b}, e_{\alpha_{2}}, 1\right)\end{array}$ & $\begin{array}{c}\emptyset \\
\left\{\alpha_{2}\right\}\end{array}$ \\
\hline$t_{c}$ & $\left\{\alpha_{2}, \alpha_{1}+\alpha_{2}\right\}$ & $\left\{\alpha_{1}\right\}$ & $\begin{array}{l}3 \\
3\end{array}$ & $\begin{array}{c}\left(t_{c},\{1\}\right) \\
\left(s_{2} t_{c},\{2\}\right)\end{array}$ & $\begin{array}{c}\left(t_{c}, 0,1\right) \\
\left(t_{c}, e_{\alpha_{2}}, 1\right)\end{array}$ & $\begin{array}{l}\mathrm{nc} \\
\mathrm{nc}\end{array}$ \\
\hline$t_{d}$ & $\left\{\alpha_{1}, \alpha_{1}+\alpha_{2}\right\}$ & $\left\{\alpha_{2}\right\}$ & $\begin{array}{l}3 \\
3\end{array}$ & $\begin{array}{c}\left(t_{d},\{2\}\right) \\
\left(s_{1} t_{d},\{1\}\right)\end{array}$ & $\begin{array}{c}\left(t_{d}, 0,1\right) \\
\left(t_{d}, e_{\alpha_{1}}, 1\right)\end{array}$ & $\begin{array}{l}\mathrm{nc} \\
\mathrm{nc}\end{array}$ \\
\hline$t_{e}$ & $\emptyset$ & $\left\{\alpha_{1}\right\}$ & 6 & $\left(t_{e},\{1\}\right)$ & $\left(t_{e}, 0,1\right)$ & nc \\
\hline$t_{f}$ & $\emptyset$ & $\left\{\alpha_{2}\right\}$ & 6 & $\left(t_{f},\{2\}\right)$ & $\left(t_{f}, 0,1\right)$ & nc \\
\hline$t_{g}$ & $\emptyset$ & $\emptyset$ & 6 & $\left(t_{g}, \emptyset\right)$ & $\left(t_{g}, 0,1\right)$ & $\emptyset$ \\
\hline$t_{o}$ & $\emptyset$ & $\left\{\alpha_{1}, \alpha_{2}\right\}$ & 6 & tempered & $\left(t_{o}, 0,1\right)$ & nc \\
\hline
\end{tabular}

Table 4.1. Irreducible representations

$\dagger$ There is one case when this representation is tempered, see Table 4.2. 
Figure 4.1 displays the real parts of the central characters in Table 4.1. If $t \in T$ then the polar decomposition $t=t_{r} t_{c}$ determines an element $\nu \in \mathbb{R}^{n}$ such that $t_{r}\left(X^{\lambda}\right)=e^{\langle\lambda, \nu\rangle}($ see $(1.3))$. For each central character $t_{p}$ the point labeled by $p$ in Figure 4.1 is the graph of the corresponding $\nu_{p} \in \mathbb{R}^{n}$. Assume (for pictorial convenience) that $q$ is a positive real number and let

$$
H_{\beta}=\left\{x \in \mathbb{R}^{n} \mid\langle\beta, x\rangle=0\right\}, \quad \text { and } \quad H_{\beta \pm \delta}=\left\{x \in \mathbb{R}^{n} \mid\langle\beta, x\rangle=\ln \left(q^{ \pm 2}\right)\right\},
$$

for each positive root $\beta$. The dotted lines display the (affine) hyperplanes $H_{\beta \pm \delta}$.

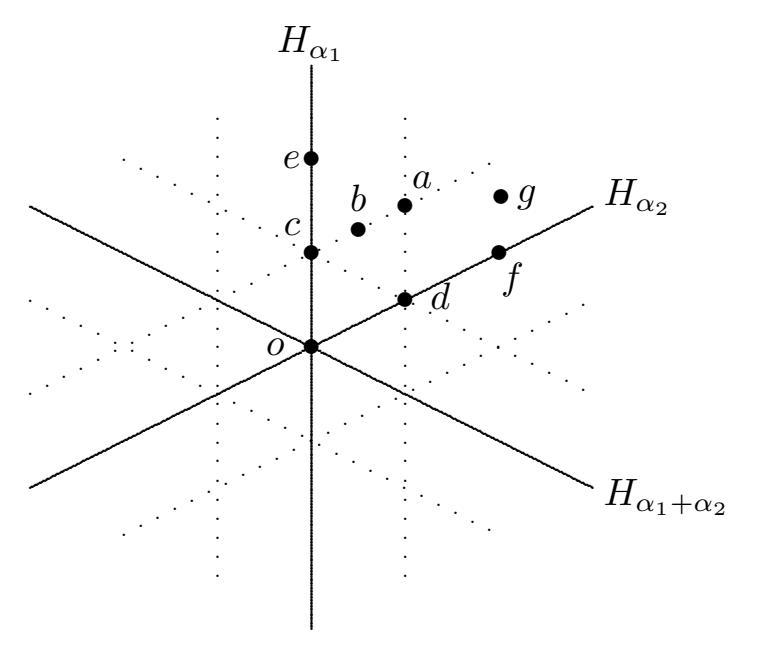

Figure 4.1. Real parts of central characters in Table 4.1

Tempered and square integrable representations. The tempered (resp. square integrable) $\tilde{H}$-modules are the ones which have the real parts of all their weights in the closure (resp. interior) of the shaded region of Figure 4.2. Let $t \in T$ be given by $t\left(X^{-\alpha_{1}}\right)= \pm q, t\left(X^{-\alpha_{2}}\right)= \pm q$. This is a special case of the central character $t_{b}$ in Table 4.1. For this particular special case there is one tempered representation with central character $t$.

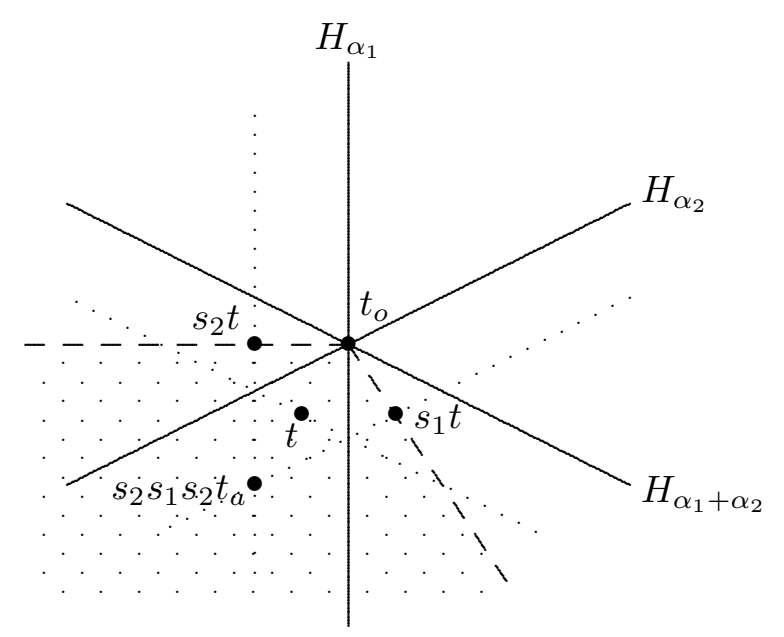

Figure 4.2. Real parts of weights of tempered representations

The irreducible tempered representations with real central character are in one-to-one correspondence with the irreducible representations of the symmetric group $S_{3}$ (see $[\mathrm{BM}]$ ). These 
representations are indexed by the partitions $(3),(21),\left(1^{3}\right)$ of 3 . Equivalently, they can be indexed by the pairs $(n, \rho)$ which appear in the Springer correspondence. The $n$ and $\rho$ will also be elements of the indexing triple for the corresponding tempered representation of $\tilde{H}$. Here $n$ is a nilpotent element of the Lie algebra $\mathfrak{g}=\mathfrak{s l}_{3}$ and $\rho$ is an irreducible representation of the component group $Z_{G}(n) / Z_{G}(n)^{\circ}$. In type $\mathrm{A}$ the component group is always trivial. For each $\operatorname{root} \beta \in R$ let $e_{\beta}$ be an element of the root space $\mathfrak{g}_{\beta}$. The three nilpotent orbits in $\mathfrak{g}$ and the corresponding tempered representations of $\tilde{H}$ are as in Table 4.2 .

$\begin{array}{ccccc}\text { Nilpotent orbit } & Z_{G}(n) / Z_{G}(n)^{\circ} & \text { Indexing triple } & \text { Square integrable } & W \text { representation } \\ \text { regular } & 1 & \left(t_{a}, e_{\alpha_{1}}+e_{\alpha_{2}}, 1\right) & \text { yes } & (3) \\ \text { subregular } & 1 & \left(s_{2} s_{1} t, e_{\alpha_{2}}, 1\right) & \text { no } & (21) \\ 0 & 1 & \left(t_{o}, 0,1\right) & \text { no } & \left(1^{3}\right)\end{array}$

Table 4.2. Tempered representations and the Springer correspondence
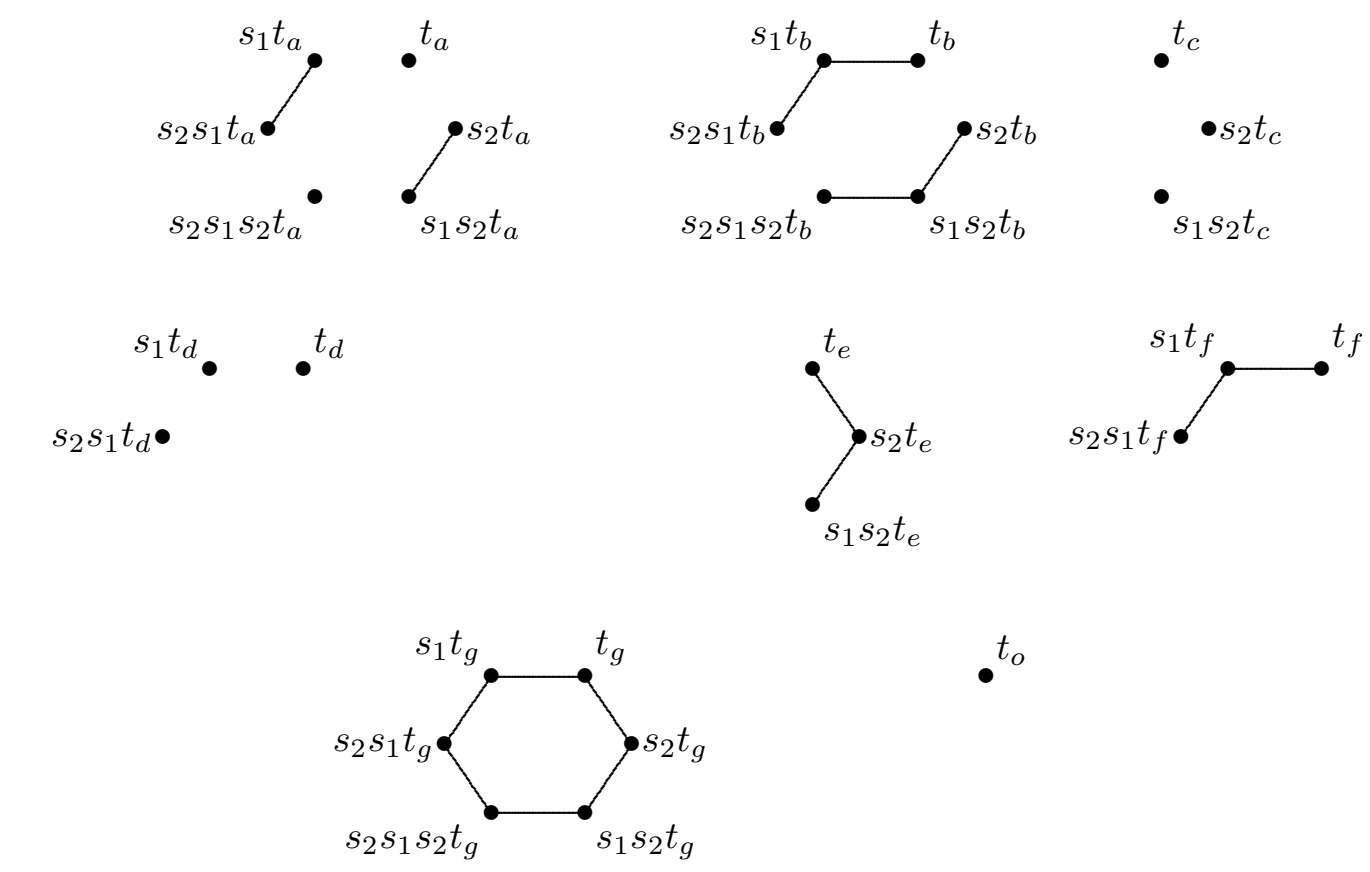

Figure 4.3. Calibration graphs for central characters in Table 4.1

\section{The analysis.}

Central characters $t_{a}, t_{b}, t_{g}$ : Since $Z(t)=\emptyset$ these weights are regular. Thus the representations corresponding to these central characters are in one to one correspondence with the connected components of the calibration graph $\Gamma(t)$ and can be constructed explicitly with the use of Theorem 1.11. Up to isomorphism the principal series module $M\left(t_{g}\right)$ is the only irreducible $\tilde{H}$-module with central character $t_{g}$. The Langlands parameters for each module can be determined from its weight structure and the indexing triple is then determined from the Langlands data by using the induction theorem of Kazhdan and Lusztig (see the discusssion in [BM, p.34]).

There is one special case of the central character $t_{b}$ when the irreducible module constructed by applying Theorem 1.11 to the placed skew shape $\left(t_{b}, \emptyset\right)$ is tempered. This happens when $t_{b}=s_{2} s_{1} t$ for the weight $t \in T$ given by $t\left(X^{-\alpha_{1}}\right)= \pm q, t\left(X^{-\alpha_{2}}\right)= \pm q$. The indexing triple and the calibration set for this case are still given by $\left(t_{b}, e_{\alpha_{2}}, 1\right)$ and $J=\left\{\alpha_{2}\right\}$, respectively. 
Central characters $t_{c}$ and $t_{d}$ : One can use the defining relations of $\tilde{H}$ to check that the only 1-dimensional representations of $\tilde{H}$ are the ones with central character $t_{a}$. Construct two 3 dimensional representations of $\tilde{H}$ by

$$
\operatorname{Ind}_{\tilde{H}_{\{2\}}^{\tilde{H}}}^{\tilde{\tilde{C}}}\left(\mathbb{C} v_{c}\right) \quad \text { and } \quad \operatorname{Ind}_{\tilde{H}_{\{2\}}^{\tilde{H}}}^{\tilde{\tilde{H}}}\left(\mathbb{C} v_{s_{2} c}\right),
$$

where $\mathbb{C} v_{c}$ and $\mathbb{C} v_{s_{2} c}$ are the two one-dimensional representations of $\tilde{H}_{\{2\}}$ given by

$$
\begin{array}{ccrl}
T_{2} v_{c}=q v_{c}, & X^{\alpha_{1}} v_{c}=v_{c}, & X^{\alpha_{2}} v_{c}=q^{2} v_{c}, \\
T_{2} v_{s_{2} c}=-q^{-1} v_{s_{2} c}, & X^{\alpha_{1}} v_{s_{2} c}=q^{2} v_{s_{2} c}, & X^{\alpha_{2}} v_{s_{2} c}=q^{-2} v_{s_{2} c} .
\end{array}
$$

These representations must be irreducible since, if not, they would either have a 1-dimensional submodule or a one dimensional quotient. But there are no 1-dimensional modules with central character $t_{c}$.

The central characters $t_{c}$ and $t_{d}$ are taken into each other under the automorphism of the Dynkin diagram of $A_{2}$ which switches the two nodes and thus these two central characters will produce modules which have the same structure (up to twisting by the automorphism which switches $\alpha_{1}$ and $\alpha_{2}$ ). Thus the representations with central character $t_{d}$ can be obtained from the ones with central character $t_{c}$ by switching all 1's and 2's and changing all $c$ 's to $d$ 's.

Central characters $t_{e}$ and $t_{f}$ : Since $P\left(t_{e}\right)=\emptyset$, Kato's irreducibility criterion (Theorem 1.16) implies that the principal series module $M\left(t_{e}\right)$ is irreducible. By Theorem 1.15 this is the only irreducible with central character $t_{e}$. As for the case of $t_{c}$ and $t_{d}$, the central characters $t_{e}$ and $t_{f}$ are taken into each other under the automorphism of the Dynkin diagram of $A_{2}$. Thus the irreducible representations with central character $t_{f}$ can be obtained from the one with central character $t_{e}$ by switching all 1's and 2's and changing all $e$ 's to $f$ 's.

Central character $t_{o}$ : Since $P\left(t_{o}\right)=\emptyset$, Kato's irreducibility criterion (Theorem 1.16) implies that the principal series module $M\left(t_{o}\right)$ is irreducible. By Theorem 1.15 this is the only irreducible with central character $t_{o}$.

\section{Classification for $C_{2}$}

The root system $R$ for $C_{2}$ has simple roots $\alpha_{1}$ and $\alpha_{2}$, fundamental weights $\omega_{1}$ and $\omega_{2}$, and

$$
\begin{array}{ll}
\left\langle\alpha_{1}, \alpha_{2}^{\vee}\right\rangle=-2 & \omega_{1}=\alpha_{1}+\alpha_{2} \\
\left\langle\alpha_{2}, \alpha_{1}^{\vee}\right\rangle=-1, & \omega_{2}=\frac{1}{2} \alpha_{1}+\alpha_{2},
\end{array} \quad \text { and } \quad \begin{aligned}
& \alpha_{1}=2 \omega_{1}-2 \omega_{2} \\
& \alpha_{2}=-\omega_{1}+2 \omega_{2} .
\end{aligned}
$$

Irreducible representations. Table 5.1 lists the irreducible $\tilde{H}$-modules by their central characters. We have listed only those central characters $t$ for which the principal series module $M(t)$ is not irreducible (see Theorem 1.16). The sets $P(t)$ and $Z(t)$ are as given in (1.7) and correspond to the choice of representative for the central character displayed in Figure 5.1. The Langlands parameters usually consist of a pair $(\mathcal{T}, I)$ where $I$ is a subset of $\{1,2\}$ and $\mathcal{T}$ is a tempered representation for the parabolic subalgebra $\tilde{H}_{I}$. In our cases the tempered representation $\mathcal{T}$ of $\tilde{H}_{I}$ is completely determined by a character $t \in T$. Specifically, $\mathcal{T}$ is the only tempered representation of $\tilde{H}_{I}$ which has $t$ as a weight. In the labeling in Table 5.1 we have replaced the representation $\mathcal{T}$ by the weight $t$. The notation for the nilpotent elements in the indexing triples is as in Table 5.2. For each calibrated module with central character $t$ we have listed the subset $J \subseteq P(t)$ such that 
$(t, J)$ is the corresponding placed skew shape (see Theorem 1.11). The abbreviation 'nc' indicates modules that are not calibrated.

\begin{tabular}{|c|c|c|c|c|c|}
\hline $\begin{array}{c}\text { Central } \\
\text { char. }\end{array}$ & $\begin{array}{l}P(t) \\
Z(t)\end{array}$ & Dim. & $\begin{array}{l}\text { Langlands } \\
\text { parameters }\end{array}$ & $\begin{array}{l}\text { Indexing } \\
\text { triple }\end{array}$ & $\begin{array}{c}\text { Calibration } \\
\text { set } J\end{array}$ \\
\hline$t_{a}$ & $\begin{array}{c}\left\{\alpha_{1}, \alpha_{2}\right\} \\
\emptyset\end{array}$ & $\begin{array}{l}1 \\
3 \\
3 \\
1\end{array}$ & $\begin{array}{l}\left(s_{1} s_{2} s_{1} s_{2} t_{a}, \emptyset\right) \\
\quad\left(s_{1} t_{a},\{1\}\right) \\
\left(s_{2} t_{a},\{2\}\right) \\
\text { tempered }\end{array}$ & $\begin{array}{c}\left(t_{a}, 0,1\right) \\
\left(t_{a}, e_{\alpha_{1}}, 1\right) \\
\left(t_{a}, e_{\alpha_{2}}, 1\right) \\
\left(t_{a}, e_{\alpha_{1}}+e_{\alpha_{2}}, 1\right)\end{array}$ & $\begin{array}{c}\emptyset \\
\left\{\alpha_{1}\right\} \\
\left\{\alpha_{2}\right\} \\
\left\{\alpha_{1}, \alpha_{2}\right\}\end{array}$ \\
\hline$t_{b}$ & $\begin{array}{c}\left\{\alpha_{1}, \alpha_{1}+\alpha_{2},\right. \\
\left.\alpha_{1}+2 \alpha_{2}\right\} \\
\left\{\alpha_{2}\right\}\end{array}$ & $\begin{array}{l}3 \\
1 \\
1 \\
3\end{array}$ & $\begin{array}{l}\left(t_{b},\{2\}\right) \\
\left(s_{1} t_{b},\{1\}\right) \\
\text { tempered } \\
\text { tempered }\end{array}$ & $\begin{array}{c}\left(t_{b}, 0,1\right) \\
\left(t_{b}, e_{\alpha_{1}}, 1\right) \\
\left(t_{b}, e_{\alpha_{1}+\alpha_{2}},-1\right) \\
\left(t_{b}, e_{\alpha_{1}+\alpha_{2}}, 1\right)\end{array}$ & $\begin{array}{c}\mathrm{nc} \\
\left\{\alpha_{1}\right\} \\
\left\{\alpha_{1}, \alpha_{1}+\alpha_{2}\right\} \\
\mathrm{nc}\end{array}$ \\
\hline$t_{c}$ & $\left\{\alpha_{1}, \alpha_{1}+2 \alpha_{2}\right\}$ & $\begin{array}{l}2 \\
2 \\
2 \\
2\end{array}$ & $\begin{array}{c}\left(t_{c},\{2\}\right) \\
\left(s_{1} t_{c},\{1\}\right) \\
\left(s_{1} s_{2} t_{c},\{1\}\right) \\
\text { tempered }\end{array}$ & $\begin{array}{c}\left(t_{c}, 0,1\right) \\
\left(t_{c}, e_{\alpha_{1}}, 1\right) \\
\left(t_{c}, e_{\alpha_{1}+2 \alpha_{2}}, 1\right) \\
\left(t_{c}, e_{\alpha_{1}}+e_{\alpha_{1}+2 \alpha_{2}}, 1\right)\end{array}$ & $\begin{array}{c}\emptyset \\
\left\{\alpha_{1}\right\} \\
\left\{\alpha_{1}+2 \alpha_{2}\right\} \\
\left\{\alpha_{1}, \alpha_{1}+2 \alpha_{2}\right\}\end{array}$ \\
\hline$t_{d}$ & $\begin{array}{c}\left\{\alpha_{2}, \alpha_{1}+\alpha_{2}\right\} \\
\left\{\alpha_{1}\right\}\end{array}$ & $\begin{array}{l}4 \\
4\end{array}$ & $\begin{array}{c}\left(t_{d},\{1\}\right) \\
\left(s_{2} t_{d},\{2\}\right)\end{array}$ & $\begin{array}{c}\left(t_{d}, 0,1\right) \\
\left(t_{d}, e_{\alpha_{2}}, 1\right)\end{array}$ & $\begin{array}{l}\mathrm{nc} \\
\mathrm{nc}\end{array}$ \\
\hline$t_{e}$ & $\begin{array}{c}\left\{\alpha_{1}\right\} \\
\left\{\alpha_{1}+2 \alpha_{2}\right\}\end{array}$ & $\begin{array}{l}4 \\
4\end{array}$ & $\begin{array}{l}\left(s_{2} t_{e},\{1\}\right) \\
\text { tempered }\end{array}$ & $\begin{array}{c}\left(t_{e}, 0,1\right) \\
\left(t_{e}, e_{\alpha_{1}}, 1\right)\end{array}$ & $\begin{array}{l}\mathrm{nc} \\
\mathrm{nc}\end{array}$ \\
\hline$t_{f}$ & $\begin{array}{c}\left\{\alpha_{1}\right\} \\
\emptyset\end{array}$ & $\begin{array}{l}4 \\
4\end{array}$ & $\begin{array}{c}\left(t_{f}, \emptyset\right) \\
\left(s_{1} t_{f},\{1\}\right)\end{array}$ & $\begin{array}{c}\left(t_{f}, 0,1\right) \\
\left(t_{f}, e_{\alpha_{1}}, 1\right)\end{array}$ & $\begin{array}{c}\emptyset \\
\left\{\alpha_{1}\right\}\end{array}$ \\
\hline$t_{g}$ & $\begin{array}{c}\left\{\alpha_{2}\right\} \\
\emptyset\end{array}$ & $\begin{array}{l}4 \\
4\end{array}$ & $\begin{array}{c}\left(t_{g}, \emptyset\right) \\
\left(s_{2} t_{g},\{2\}\right)\end{array}$ & $\begin{array}{c}\left(t_{g}, 0,1\right) \\
\left(t_{g}, e_{\alpha_{2}}, 1\right)\end{array}$ & $\begin{array}{c}\emptyset \\
\left\{\alpha_{2}\right\}\end{array}$ \\
\hline
\end{tabular}

Table 5.1. Irreducible (non principal series) representations

Figure 5.1 displays the real parts of the central characters in Table 5.1. If $t \in T$ then the polar decomposition $t=t_{r} t_{c}$ determines an element $\mu \in \mathbb{R}^{n}$ such that $t_{r}\left(X^{\lambda}\right)=e^{\langle\lambda, \mu\rangle}$ (see (1.3)). For each central character $t_{p}$ the point labeled by $p$ in Figure 5.1 is the graph of the corresponding $\mu_{p} \in \mathbb{R}^{n}$. Assume (for pictorial convenience) that $q$ is a positive real number and let

$$
H_{\beta}=\left\{x \in \mathbb{R}^{n} \mid\langle\beta, x\rangle=0\right\}, \quad \text { and } \quad H_{\beta \pm \delta}=\left\{x \in \mathbb{R}^{n} \mid\langle\beta, x\rangle=\ln \left(q^{ \pm 2}\right)\right\}
$$

for each positive root $\beta$. The dotted lines display the (affine) hyperplanes $H_{\beta \pm \delta}$. 


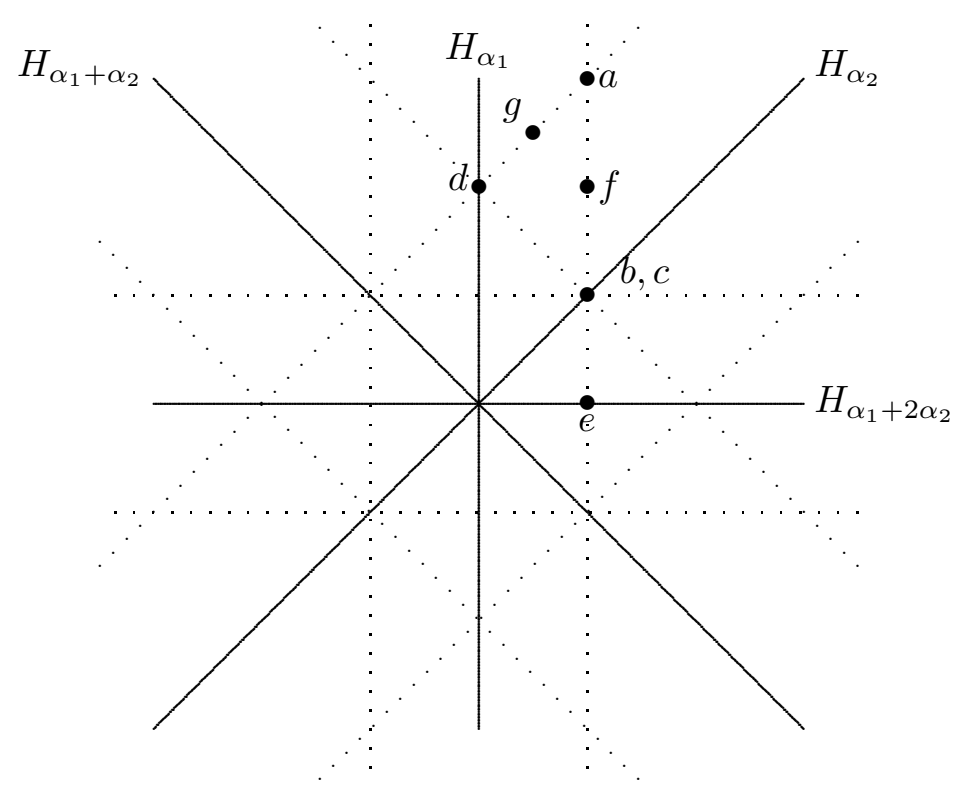

Figure 5.1. Real parts of central characters in Table 5.1

Tempered and square integrable representations. The tempered (resp. square integrable) $\tilde{H}$-modules are the ones which have the real parts of all their weights in the closure (resp. interior) of the shaded region of Figure 5.2.

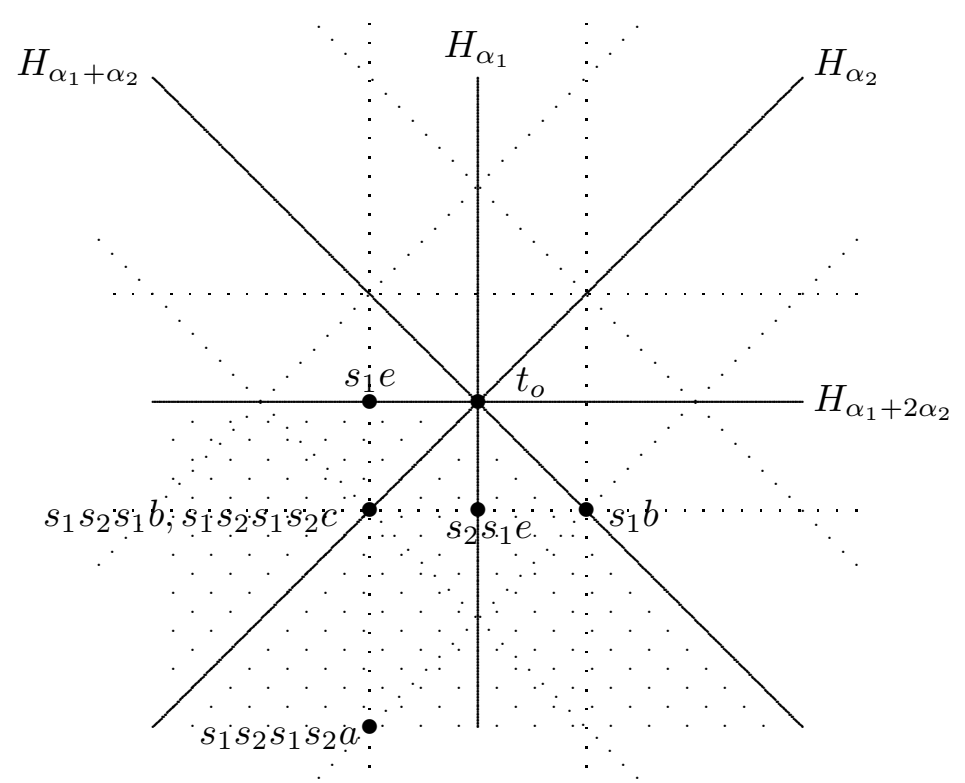

Figure 5.2. Real parts of weights of tempered representations 
The irreducible tempered representations with real central character can be indexed by the irreducible representations of the Weyl group $W$ of type $C_{2}$ (see [BM, p. 34]). Equivalently, these representations can be indexed by the pairs $(n, \rho)$ which appear in the Springer correspondence. The $n$ and $\rho$ will also be elements of the indexing triple for the corresponding tempered representation of $\tilde{H}$. Here $n$ is a nilpotent element of the Lie algebra $\mathfrak{g}=\operatorname{Lie}(G), G$ is the complex simple group over $\mathbb{C}$ of type $C_{2}$ and $\rho$ is an irreducible representation of the component group $Z_{G}(n) / Z_{G}(n)^{\circ}$ (see $[\mathrm{Ca}]$ ). For each $\operatorname{root} \beta \in R$ let $e_{\beta}$ be an element of the root space $\mathfrak{g}_{\beta}$. The four nilpotent orbits in $\mathfrak{g}$ and the corresponding tempered representations of $\tilde{H}$ are as in Table 5.2. We have used the notation of Carter [Ca, p.424] to label the irreducible representations of the Weyl group.

$\begin{array}{ccccc}\text { Nilpotent orbit } & Z_{G}(n) / Z_{G}(n)^{\circ} & \text { Indexing triple } & \text { Square integrable } & W \text { representation } \\ \text { regular } & 1 & \left(t_{a}, e_{\alpha_{1}}+e_{\alpha_{2}}, 1\right) & \text { yes } & (\emptyset, 11) \\ \text { subregular } & \mathbb{Z} / 2 \mathbb{Z} & \left(t_{b}, e_{\alpha_{1}+\alpha_{2}}, 1\right) & \text { yes } & (1,1) \\ & & \left(t_{b}, e_{\alpha_{1}+\alpha_{2}},-1\right) & \text { yes } & (\emptyset, 2) \\ \text { minimal } & 1 & \left(t_{e}, e_{\alpha_{1}}, 1\right) & \text { no } & (11, \emptyset) \\ 0 & 1 & \left(t_{o}, 0,1\right) & \text { no } & (2, \emptyset)\end{array}$

Table 5.2. Tempered representations and the Springer correspondence

The only other tempered representation is the square integrable representation $\left(t_{c}, e_{\alpha_{1}}+e_{\alpha_{1}+2 \alpha_{2}}, 1\right)$. This representation does not have real central character. It is the representation constructed in [Lu3, 4.14, 4.23]. (In Lusztig's notation it is the star of the representation corresponding to the graph $\mathcal{G}^{\prime} \oplus \mathcal{G}^{\prime \prime}$.)
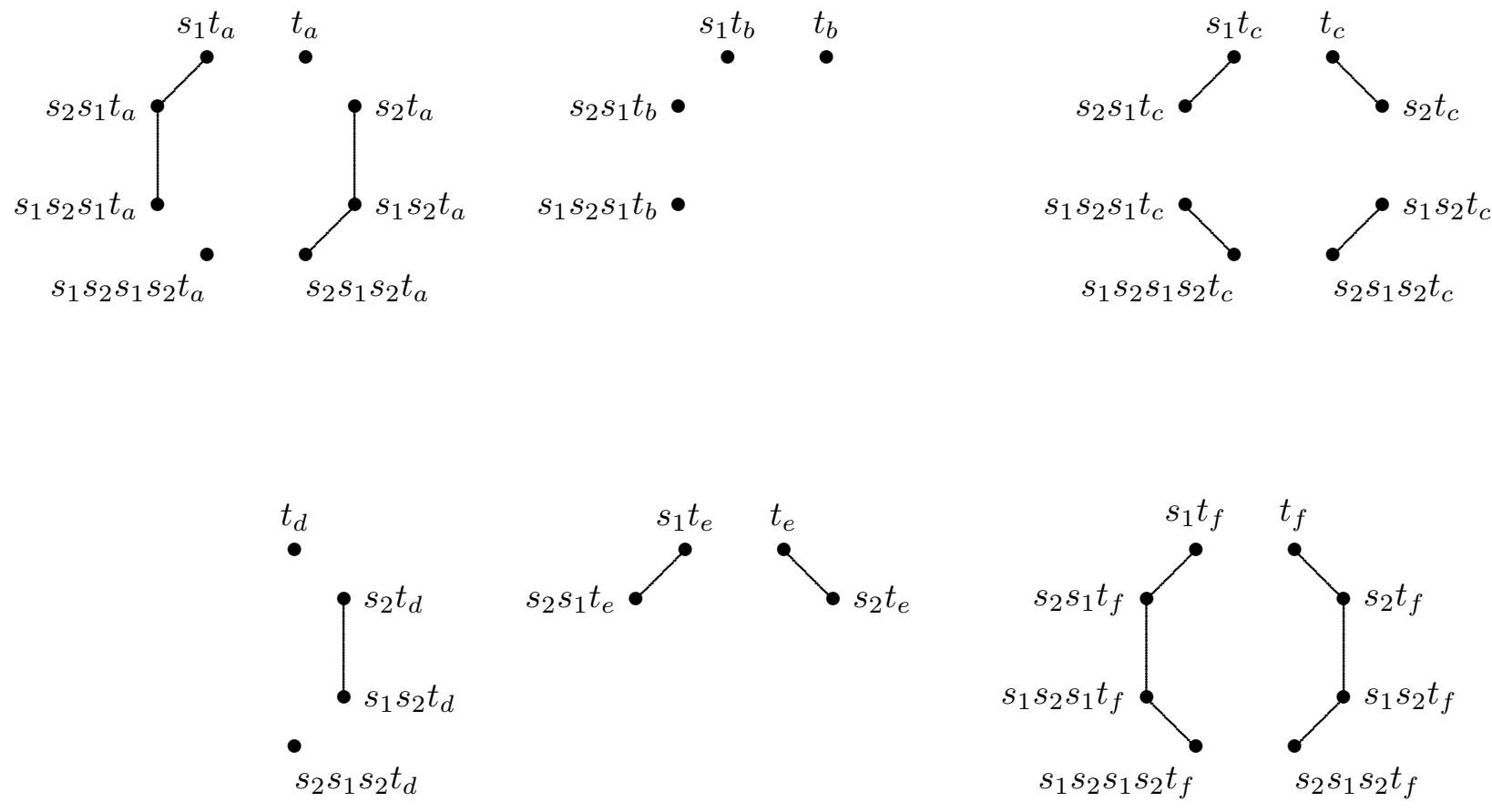


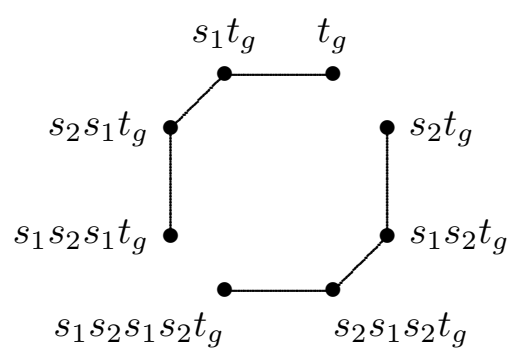

Figure 5.3. Calibration graphs for central characters in Table 5.1

The analysis. A general calculation with the defining relations of $\tilde{H}$ shows that there are only four one dimensional $\tilde{H}$-modules $\mathbb{C} v_{1}, \mathbb{C} v_{2}, \mathbb{C} v_{3}$, and $\mathbb{C} v_{4}$ which have weights $t_{a}, s_{2} s_{1} t_{b}, s_{1} t_{b}$ and $s_{1} s_{2} s_{1} s_{2} t_{a}$, respectively. These modules are given explicitly by

$$
\begin{array}{cccc}
T_{1} v_{1}=q v_{1}, & T_{2} v_{1}=q v_{1}, & X^{\alpha_{1}} v_{1}=q^{2} v_{1}, & X^{\alpha_{2}} v_{1}=q^{2} v_{1}, \\
T_{1} v_{2}=q v_{2}, & T_{2} v_{2}=-q^{-1} v_{2}, & X^{\alpha_{1}} v_{2}=q^{2} v_{2}, & X^{\alpha_{2}} v_{2}=q^{-2} v_{2}, \\
T_{1} v_{3}=-q^{-1} v_{3}, & T_{2} v_{3}=q v_{3}, & X^{\alpha_{1}} v_{3}=q^{-2} v_{3}, & X^{\alpha_{2}} v_{3}=q^{2} v_{3}, \\
T_{1} v_{4}=-q^{-1} v_{4}, & T_{2} v_{4}=-q^{-1} v_{4}, & X^{\alpha_{1}} v_{4}=q^{-2} v_{4}, & X^{\alpha_{2}} v_{4}=q^{-2} v_{4} .
\end{array}
$$

Central character $t_{a}$ : Since $Z\left(t_{a}\right)=\emptyset, t_{a}$ is regular and thus all irreducible representations with central character $t_{a}$ are calibrated. They are in one to one correspondence with the connected components of the calibration graph and can be constructed with the use of Theorem 1.11. The Langlands parameters for each module can be determined from its weight structure and the indexing triple is then determined from the Langlands data by using the induction theorem of Kazhdan-Lusztig (see the discussion in [BM, p.34]).

Central character $t_{b}$ : We already know from our general computation above, that there are two one-dimensional $\tilde{H}$-modules with central character $t_{b}$. One has weight $s_{1} t_{b}$ and the other has weight $s_{2} s_{1} t_{b}$. Let $\mathbb{C} v_{b}$ and $\mathbb{C} v_{s_{1} b}$ be the one dimensional representations of $\tilde{H}_{\{1\}}$ given by

$$
\begin{array}{ccc}
T_{1} v_{b}=q v_{b}, & X^{\alpha_{1}} v_{b}=q^{2} v_{b}, & X^{\alpha_{2}} v_{b}=v_{b}, \\
T_{1} v_{s_{1} b}=-q^{-1} v_{s_{1} b}, & X^{\alpha_{1}} v_{s_{1} b}=q^{-2} v_{s_{1} b}, & X^{\alpha_{2}} v_{s_{1} b}=q^{2} v_{s_{1} b} .
\end{array}
$$

Let

$$
M_{1}=\operatorname{Ind}_{\tilde{H}_{\{1\}}}^{\tilde{H}}\left(\mathbb{C} v_{b}\right) \quad \text { and } \quad M_{2}=\operatorname{Ind}_{\tilde{H}_{\{1\}}}^{\tilde{\tilde{H}}}\left(\mathbb{C} v_{s_{1} b}\right)
$$

By Lemma 1.17 these modules have weights $\operatorname{supp}\left(M_{1}\right)=\left\{t_{b}, s_{1} t_{b}, s_{2} s_{1} t_{b}\right\}$ and $\operatorname{supp}\left(M_{2}\right)=\left\{s_{1} t_{b}\right.$, $\left.s_{2} s_{1} t_{b}, s_{1} s_{2} s_{1} t_{b}\right\}$, respectively. Both $M_{1}$ and $M_{2}$ are 4 dimensional. By Proposition 1.18 (c) one of the two operators $\tau_{2}:\left(M_{1}\right)_{s_{2} s_{1} t_{b}} \rightarrow\left(M_{1}\right)_{s_{1} t_{b}}$ or $\tau_{2}:\left(M_{1}\right)_{s_{1} t_{b}} \rightarrow\left(M_{1}\right)_{s_{2} s_{1} t_{b}}$ must have nonzero kernel. This implies that $M_{1}$ has either a 3 dimensional submodule or a 3 dimensional quotient, call it $N_{1}$, with weights $\left\{t_{b}, s_{1} t_{b}\right\}$. By Lemma 1.19 , any module $P$ with weights $\left\{t_{b}, s_{1} t_{b}\right\}$ must have $\operatorname{dim}\left(P_{t_{b}}^{\text {gen }}\right) \geq 2$ and $\operatorname{dim}\left(P_{s_{1} t_{b}}^{\text {gen }}\right) \geq 1$. It follows that $N_{1}$ is irreducible. A similar argument can be used to show that $M_{2}$ has either a 3 dimensional submodule or a 3-dimensional quotient which must be irreducible.

The representation $N_{1}$ constructed in the previous paragraph and the 1 dimensional representation with weight $s_{1} t_{b}$ are both tempered. One obtains the corresponding indexing triples by comparing the Langlands parameters for these modules with the labelings of the corresponding representations of $W$ in the Springer correspondence. See [Ca, p.424], [BM, p. 34] and Table 5.2. 
Central character $t_{c}$ : Since $Z\left(t_{c}\right)=\emptyset, t_{c}$ is regular and thus all irreducible representations with central character $t_{c}$ are calibrated. They are in one to one correspondence with the connected components of the calibration graph and can be constructed with the use of Theorem 1.11. The Langlands parameters for each module can be determined from its weight structure. The only representation for which the indexing triple cannot be determined from the Langlands parameters and the $[\mathrm{KL}]$ induction theorem (see the discussion in [BM, p. 34]) is the tempered representation. This representation is constructed in [Lu3, 4.14 and 4.23]. In Lusztig's notation, it is the star (see $[\mathrm{Lu} 3,4.23])$ of the representation corresponding to the graph $\mathcal{G}^{\prime} \oplus \mathcal{G}^{\prime \prime}$. The indexing triple for this representation is given in the discussion for $B_{2}$ in [Lu3, 2.10].

Central character $t_{d}$ : Let $\mathbb{C} v_{d}$ and $\mathbb{C} v_{s_{2} d}$ be the one dimensional representations of $\tilde{H}_{\{2\}}$ given by

$$
\begin{array}{ccc}
T_{2} v_{d}=q v_{d}, & X^{\alpha_{1}} v_{d}=v_{d}, & X^{\alpha_{2}} v_{d}=q^{2} v_{d}, \\
T_{2} v_{s_{2} d}=-q^{-1} v_{s_{2} d}, & X^{\alpha_{1}} v_{s_{2} d}=q^{4} v_{s_{2} d}, & X^{\alpha_{2}} v_{s_{2} d}=q^{-2} v_{s_{2} d} .
\end{array}
$$

Let

$$
M_{1}=\operatorname{Ind}_{\tilde{H}_{\{2\}}}^{\tilde{H}}\left(\mathbb{C} v_{d}\right) \quad \text { and } \quad M_{2}=\operatorname{Ind}_{\tilde{H}_{\{2\}}}^{\tilde{\tilde{H}}}\left(\mathbb{C} v_{s_{2} d}\right) .
$$

By Lemma 1.17 these modules have weights $\operatorname{supp}\left(M_{1}\right)=\left\{t_{d}, s_{2} t_{d}, s_{1} s_{2} t_{d}\right\}$ and $\operatorname{supp}\left(M_{2}\right)=$ $\left\{s_{2} t_{d}, s_{1} s_{2} t_{d}, s_{2} s_{1} s_{2} t_{d}\right\}$, respectively. Both $M_{1}$ and $M_{2}$ are 4 dimensional.

Let $M$ be any $\tilde{H}$-module such that $M_{t_{d}} \neq 0$. By Lemma $1.19(\mathrm{a}), \operatorname{dim}\left(M_{t_{d}}^{\text {gen }}\right) \geq 2$. Since $\left\langle\alpha_{2}, \alpha_{1}^{\vee}\right\rangle \neq 0$ it follows from Lemma 1.19 (b) that $\operatorname{dim}\left(M_{s_{2} t_{d}}^{\mathrm{gen}}\right) \geq 1$. Then, by Proposition 1.6, $\operatorname{dim}\left(M_{s_{1} s_{2} t_{d}}^{\text {gen }}\right) \geq 1$. Adding these numbers up we see that $\operatorname{dim}(M) \geq 4$. It follows that $M_{1}$ is irreducible. An analogous argument can be applied to conclude that $M_{2}$ is irreducible.

Central character $t_{e}$ : Let $\mathbb{C} v_{e}$ and $\mathbb{C} v_{s_{1} e}$ be the one dimensional representations of $\tilde{H}_{\{1\}}$ given by

$$
\begin{array}{ccc}
T_{1} v_{e}=q v_{e}, & X^{\alpha_{1}} v_{e}=q^{2} v_{e}, & X^{\alpha_{2}} v_{e}=q^{-1} v_{e} \\
T_{1} v_{s_{1} e}=-q^{-1} v_{s_{1} e}, & X^{\alpha_{1}} v_{s_{1} e}=q^{-2} v_{s_{1} e}, & X^{\alpha_{2}} v_{s_{1} e}=q v_{s_{1} e}
\end{array}
$$

Let

$$
M_{1}=\operatorname{Ind}_{\tilde{H}_{\{1\}}}^{\tilde{\tilde{H}}}\left(\mathbb{C} v_{e}\right) \quad \text { and } \quad M_{2}=\operatorname{Ind}_{\tilde{H}_{\{1\}}}^{\tilde{\tilde{H}}}\left(\mathbb{C} v_{s_{1} e}\right)
$$

By Lemma 1.17 these modules have weights $\operatorname{supp}\left(M_{1}\right)=\left\{t_{e}, s_{2} t_{e}\right\}$ and $\operatorname{supp}\left(M_{2}\right)=\left\{s_{1} t_{e}, s_{2} s_{1} t_{e}\right\}$ respectively. Both $M_{1}$ and $M_{2}$ are 4 dimensional.

Let $M$ be any $\tilde{H}$-module such that $M_{t_{e}} \neq 0$. By Lemma 1.19 (a) and Proposition 1.6, $\operatorname{dim}\left(M_{t_{e}}^{\text {gen }}\right)=\operatorname{dim}\left(M_{s_{2} t_{e}}^{\text {gen }}\right) \geq 2$. Thus $\operatorname{dim}(M) \geq 4$. It follows that $M_{1}$ is irreducible. An analogous argument can be applied to conclude that $M_{2}$ is irreducible.

Central character $t_{f}$ and $t_{g}$ : These cases are handled in the same way as for the central character $t_{a}$.

\section{Classification for $G_{2}$}

The root system $R$ for $G_{2}$ has simple roots $\alpha_{1}$ and $\alpha_{2}$, fundamental weights $\omega_{1}$ and $\omega_{2}$, and

$$
\begin{array}{ll}
\left\langle\alpha_{1}, \alpha_{2}^{\vee}\right\rangle=-3 & \omega_{1}=2 \alpha_{1}+3 \alpha_{2} \\
\left\langle\alpha_{2}, \alpha_{1}^{\vee}\right\rangle=-1, & \omega_{2}=\alpha_{1}+2 \alpha_{2},
\end{array} \quad \text { and } \quad \begin{aligned}
& \alpha_{1}=-\omega_{1}+2 \omega_{2} \\
& \alpha_{2}=2 \omega_{1}-3 \omega_{2} .
\end{aligned}
$$


Irreducible representations.

\begin{tabular}{|c|c|c|c|c|}
\hline $\begin{array}{l}\text { Central } \\
\text { character }\end{array}$ & $\begin{array}{l}P(t) \\
Z(t)\end{array}$ & Dimension & $\begin{array}{l}\text { Langlands } \\
\text { parameters }\end{array}$ & $\begin{array}{l}\text { Indexing } \\
\text { triple }\end{array}$ \\
\hline$t_{a}$ & $\begin{array}{c}\left\{\alpha_{1}, \alpha_{2}\right\} \\
\emptyset\end{array}$ & $\begin{array}{l}1 \\
5 \\
5 \\
1\end{array}$ & $\begin{array}{c}\left(t_{a}, \emptyset\right) \\
\left(s_{1} t_{a},\{1\}\right) \\
\left(s_{2} t_{a},\{2\}\right) \\
\text { tempered }\end{array}$ & $\begin{array}{c}\left(t_{a}, 0,1\right) \\
\left(t_{a}, e_{\alpha_{1}}, 1\right) \\
\left(t_{a}, e_{\alpha_{2}}, 1\right) \\
\left(t_{a}, e_{\alpha_{1}}+e_{\alpha_{2}}, 1\right)\end{array}$ \\
\hline$t_{b}$ & $\begin{array}{c}\left\{\alpha_{1}\right\} \\
\emptyset\end{array}$ & $\begin{array}{l}6 \\
6\end{array}$ & $\begin{array}{c}\left(t_{b}, \emptyset\right) \\
\left(s_{1} t_{b},\{1\}\right)\end{array}$ & $\begin{array}{c}\left(t_{b}, 0,1\right) \\
\left(t_{b}, e_{\alpha_{1}}, 1\right)\end{array}$ \\
\hline$t_{c}$ & $\left\{\begin{array}{c}\left\{\alpha_{1}, \alpha_{1}+3 \alpha_{2}\right\} \\
\emptyset\end{array}\right.$ & $\begin{array}{l}2 \\
4 \\
4 \\
2\end{array}$ & $\begin{array}{c}\left(t_{c},\{2\}\right) \\
\left(s_{1} t_{c},\{1\}\right) \\
\left(s_{1} s_{2} t_{c},\{1\}\right) \\
\text { tempered }\end{array}$ & $\begin{array}{c}\left(t_{c}, 0,1\right) \\
\left(t_{c}, e_{\alpha_{1}}, 1\right) \\
\left(t_{c}, e_{\alpha_{1}+3 \alpha_{2}}, 1\right) \\
\left(t_{c}, e_{\alpha_{1}}+e_{\alpha_{1}+3 \alpha_{2}}, 1\right)\end{array}$ \\
\hline$t_{d}$ & $\left\{\begin{array}{c}\left.\alpha_{1}, \alpha_{1}+2 \alpha_{2}\right\} \\
\emptyset\end{array}\right.$ & $\begin{array}{l}3 \\
3 \\
3 \\
3\end{array}$ & $\begin{array}{c}\left(t_{d},\{2\}\right) \\
\left(s_{1} t_{d},\{1\}\right) \\
\left(s_{2} s_{1} s_{2} t_{d},\{2\}\right) \\
\text { tempered }\end{array}$ & $\begin{array}{c}\left(t_{d}, 0,1\right) \\
\left(t_{d}, e_{\alpha_{1}}, 1\right) \\
\left(t_{d}, e_{\alpha_{1}+2 \alpha_{2}}, 1\right) \\
\left(t_{d}, e_{\alpha_{1}}+e_{\alpha_{1}+2 \alpha_{2}}, 1\right)\end{array}$ \\
\hline$t_{e}$ & $\begin{array}{c}\left\{\alpha_{1}, \alpha_{1}+2 \alpha_{2},\right. \\
\left.\alpha_{1}+\alpha_{2}, \alpha_{1}+3 \alpha_{2}\right\} \\
\left\{\alpha_{2}\right\}\end{array}$ & $\begin{array}{l}3 \\
1 \\
2 \\
1 \\
3\end{array}$ & $\begin{array}{c}\left(t_{e},\{2\}\right) \\
\left(s_{1} t_{e},\{1\}\right) \\
\left(s_{2} s_{1} t_{e},\{2\}\right) \\
\text { tempered } \\
\text { tempered }\end{array}$ & $\begin{array}{c}\left(t_{e}, 0,1\right) \\
\left(t_{e}, e_{\alpha_{1}}, 1\right) \\
\left(t_{e}, e_{\alpha_{1}+\alpha_{2}}, 1\right) \\
\left(t_{e}, e_{\alpha_{1}}+e_{\alpha_{1}+2 \alpha_{2}},(21)\right) \\
\left(t_{e}, e_{\alpha_{1}}+e_{\alpha_{1}+2 \alpha_{2}},(3)\right)\end{array}$ \\
\hline$t_{f}$ & $\begin{array}{c}\left\{\alpha_{1}, 2 \alpha_{1}+3 \alpha_{2}\right\} \\
\left\{\alpha_{1}+3 \alpha_{2}\right\}\end{array}$ & $\begin{array}{l}6 \\
6\end{array}$ & $\begin{array}{c}\left(t_{f},\{1\}\right) \\
\left(s_{1} t_{f},\{1\}\right)\end{array}$ & $\begin{array}{c}\left(t_{f}, 0,1\right) \\
\left(t_{f}, e_{\alpha_{1}}, 1\right)\end{array}$ \\
\hline$t_{g}$ & $\begin{array}{c}\left\{\alpha_{1}\right\} \\
\left\{\alpha_{1}+2 \alpha_{2}\right\}\end{array}$ & $\begin{array}{l}6 \\
6\end{array}$ & $\begin{array}{l}\left(t_{g},\{2\}\right) \\
\text { tempered }\end{array}$ & $\begin{array}{c}\left(t_{g}, 0,1\right) \\
\left(t_{g}, e_{\alpha_{1}}, 1\right)\end{array}$ \\
\hline$t_{h}$ & $\left\{\alpha_{2}\right\}$ & $\begin{array}{l}6 \\
6\end{array}$ & $\begin{array}{c}\left(t_{h}, \emptyset\right) \\
\left(s_{2} t_{h},\{2\}\right)\end{array}$ & $\begin{array}{c}\left(t_{h}, 0,1\right) \\
\left(t_{h}, e_{\alpha_{2}}, 1\right)\end{array}$ \\
\hline$t_{i}$ & $\begin{array}{c}\left\{\alpha_{2}, \alpha_{1}+\alpha_{2}\right\} \\
\left\{\alpha_{1}\right\}\end{array}$ & $\begin{array}{l}6 \\
6\end{array}$ & $\begin{array}{c}\left(t_{i},\{1\}\right) \\
\left(s_{2} t_{i},\{2\}\right)\end{array}$ & $\begin{array}{c}\left(t_{i}, 0,1\right) \\
\left(t_{i}, e_{\alpha_{2}}, 1\right)\end{array}$ \\
\hline$t_{j}$ & $\begin{array}{c}\left\{\alpha_{2}\right\} \\
\left\{2 \alpha_{1}+3 \alpha_{2}\right\}\end{array}$ & $\begin{array}{l}6 \\
6\end{array}$ & $\begin{array}{c}\left(t_{j},\{1\}\right) \\
\text { tempered }\end{array}$ & $\begin{array}{c}\left(t_{j}, 0,1\right) \\
\left(t_{j}, e_{\alpha_{2}}, 1\right)\end{array}$ \\
\hline
\end{tabular}

Table 6.1. Irreducible (non principal series) representations

Table 6.1 lists the irreducible $\tilde{H}$-modules by their central characters. We have listed only those central characters $t$ for which the principal series module $M(t)$ is not irreducible (see Theorem 
1.16). The sets $P(t)$ and $Z(t)$ are as given in (1.7) and correspond to the choice of representative for the central character displayed in Figure 6.1. The Langlands parameters usually consist of a pair $(\mathcal{T}, I)$ where $I$ is a subset of $\{1,2\}$ and $\mathcal{T}$ is a tempered representation for the parabolic subalgebra $\tilde{H}_{I}$. In our cases the tempered representation $\mathcal{T}$ of $\tilde{H}_{I}$ is completely determined by a character $t \in T$. Specifically, $\mathcal{T}$ is the only tempered representation of $\tilde{H}_{I}$ which has $t$ as a weight. In the labeling in Table 6.1 we have replaced the representation $\mathcal{T}$ by the weight $t$. The notation for the nilpotent elements in the indexing triples is as in Table 6.3.

Table 6.2 lists the irreducible calibrated $\tilde{H}$-modules. For each module with central character $t$ we have listed the subset $J \subseteq P(t)$ such that $(t, J)$ is the corresponding placed skew shape (see Theorem 1.11). We have listed only those central characters $t$ for which the principal series module $M(t)$ is not irreducible (see Theorem 1.16).

\begin{tabular}{|c|c|c|c|c|c|}
\hline $\begin{array}{l}\text { Central } \\
\text { character }\end{array}$ & $P(t)$ & $Z(t)$ & Dimension & $\begin{array}{l}\text { Indexing } \\
\text { triple }\end{array}$ & $\begin{array}{c}\text { Calibration } \\
\text { set } J\end{array}$ \\
\hline$t_{a}$ & $\left\{\alpha_{1}, \alpha_{2}\right\}$ & $\emptyset$ & $\begin{array}{l}1 \\
5 \\
5 \\
1\end{array}$ & $\begin{array}{c}\left(t_{a}, 0,1\right) \\
\left(t_{a}, e_{\alpha_{1}}, 1\right) \\
\left(t_{a}, e_{\alpha_{2}}, 1\right) \\
\left(t_{a}, e_{\alpha_{1}}+e_{\alpha_{2}}, 1\right)\end{array}$ & $\begin{array}{c}\emptyset \\
\left\{\alpha_{1}\right\} \\
\left\{\alpha_{2}\right\} \\
\left\{\alpha_{1}, \alpha_{2}\right\}\end{array}$ \\
\hline$t_{b}$ & $\left\{\alpha_{1}\right\}$ & $\emptyset$ & $\begin{array}{l}6 \\
6\end{array}$ & $\begin{array}{c}\left(t_{b}, 0,1\right) \\
\left(t_{b}, e_{\alpha_{1}}, 1\right)\end{array}$ & $\begin{array}{c}\emptyset \\
\left\{\alpha_{1}\right\}\end{array}$ \\
\hline$t_{c}$ & $\left\{\alpha_{1}, \alpha_{1}+3 \alpha_{2}\right\}$ & $\emptyset$ & $\begin{array}{l}2 \\
4 \\
4 \\
2\end{array}$ & $\begin{array}{c}\left(t_{c}, 0,1\right) \\
\left(t_{c}, e_{\alpha_{1}}, 1\right) \\
\left(t_{c}, e_{\alpha_{1}+3 \alpha_{2}}, 1\right) \\
\left(t_{c}, e_{\alpha_{1}}+e_{\alpha_{1}+3 \alpha_{2}}, 1\right)\end{array}$ & $\begin{array}{c}\emptyset \\
\left\{\alpha_{1}\right\} \\
\left\{\alpha_{1}+3 \alpha_{2}\right\} \\
\left\{\alpha_{1}, \alpha_{1}+3 \alpha_{2}\right\}\end{array}$ \\
\hline$t_{d}$ & $\left\{\alpha_{1}, \alpha_{1}+2 \alpha_{2}\right\}$ & $\emptyset$ & $\begin{array}{l}3 \\
3 \\
3 \\
3\end{array}$ & $\begin{array}{c}\left(t_{d}, 0,1\right) \\
\left(t_{d}, e_{\alpha_{1}}, 1\right) \\
\left(t_{d}, e_{\alpha_{1}+2 \alpha_{2}}, 1\right) \\
\left(t_{d}, e_{\alpha_{1}}+e_{\alpha_{1}+2 \alpha_{2}}, 1\right)\end{array}$ & $\begin{array}{c}\emptyset \\
\left\{\alpha_{1}\right\} \\
\left\{\alpha_{1}+2 \alpha_{2}\right\} \\
\left\{\alpha_{1}, \alpha_{1}+2 \alpha_{2}\right\}\end{array}$ \\
\hline$t_{e}$ & $\begin{array}{l}\left\{\alpha_{1}, \alpha_{1}+2 \alpha_{2}\right. \\
\left.\alpha_{1}+\alpha_{2}, \alpha_{1}+3 \alpha_{2}\right\}\end{array}$ & $\left\{\alpha_{2}\right\}$ & $\begin{array}{l}1 \\
2 \\
1\end{array}$ & $\begin{array}{c}\left(t_{e}, e_{\alpha_{1}}, 1\right) \\
\left(t_{e}, e_{\alpha_{1}+\alpha_{2}}, 1\right) \\
\left(t_{e}, e_{\alpha_{1}}+e_{\alpha_{1}+2 \alpha_{2}},(21)\right)\end{array}$ & $\begin{array}{c}\left\{\alpha_{1}\right\} \\
\left\{\alpha_{1}, \alpha_{1}+\alpha_{2}\right\} \\
P\left(t_{e}\right) \backslash\left\{\alpha_{1}+3 \alpha_{2}\right\}\end{array}$ \\
\hline$t_{h}$ & $\left\{\alpha_{2}\right\}$ & $\emptyset$ & $\begin{array}{l}6 \\
6\end{array}$ & $\begin{array}{c}\left(t_{h}, 0,1\right) \\
\left(t_{h}, e_{\alpha_{2}}, 1\right)\end{array}$ & $\begin{array}{c}\emptyset \\
\left\{\alpha_{2}\right\}\end{array}$ \\
\hline
\end{tabular}

Table 6.2. Calibrated irreducible (non principal series) representations

Figure 6.1 displays the real parts of the central characters in Table 6.1. If $t \in T$ then the polar decomposition $t=t_{r} t_{c}$ determines an element $\nu \in \mathbb{R}^{n}$ such that $t_{r}\left(X^{\lambda}\right)=e^{\langle\nu, \lambda\rangle}($ see $(1.3)$ ). For each central character $t_{p}$ the point labeled by $p$ in Figure 6.1 is the graph of the corresponding $\nu_{p} \in \mathbb{R}^{n}$. Assume (for pictorial convenience) that $q$ is a positive real number and let

$$
H_{\beta}=\left\{x \in \mathbb{R}^{n} \mid\langle\beta, x\rangle=0\right\}, \quad \text { and } \quad H_{\beta \pm \delta}=\left\{x \in \mathbb{R}^{n} \mid\langle\beta, x\rangle=\ln \left(q^{ \pm 2}\right)\right\},
$$

for each positive root $\beta$. The dotted lines display the (affine) hyperplanes $H_{\beta \pm \delta}$. 


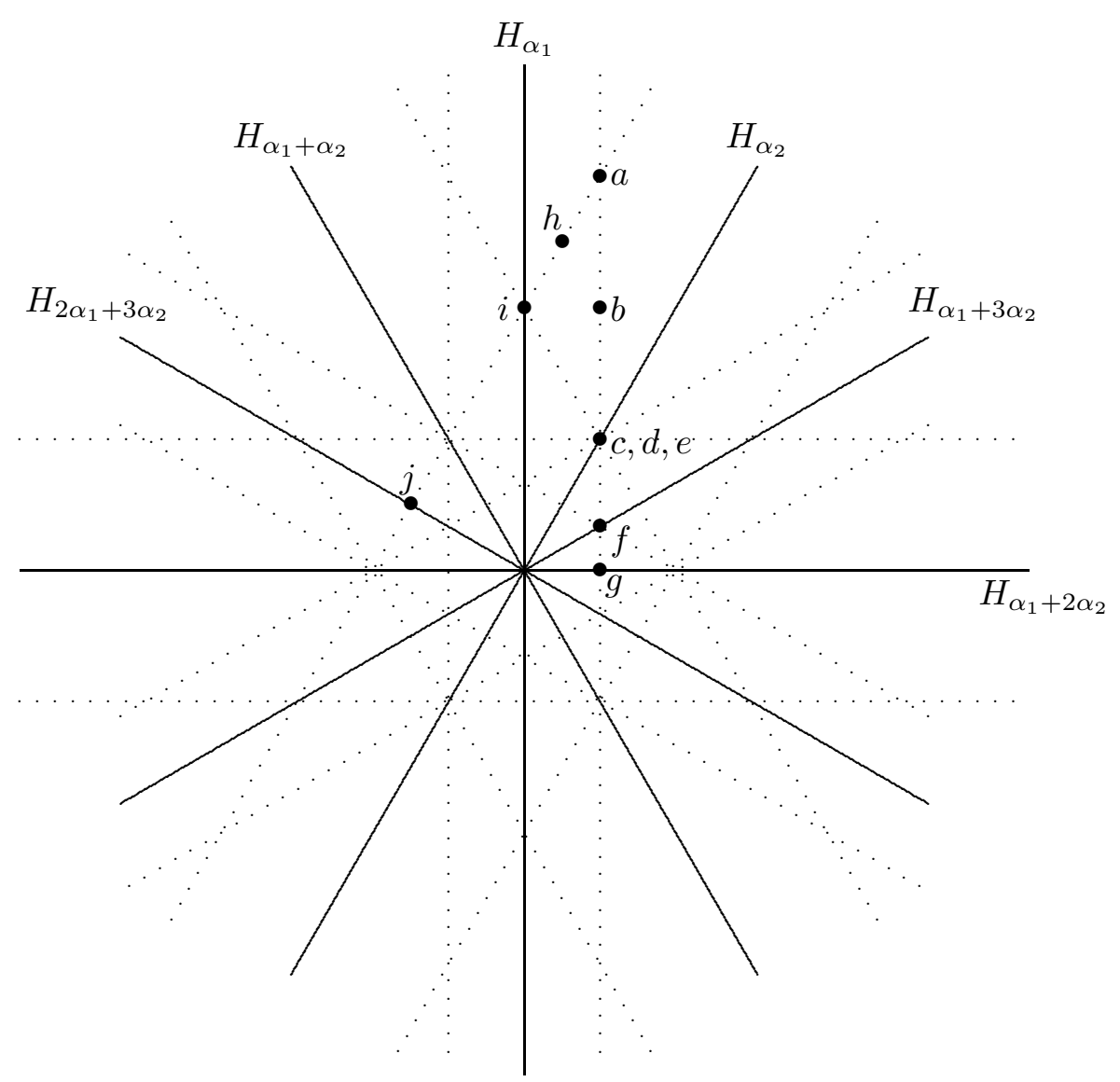

Figure 6.1. Real parts of central characters in Table 6.1

Tempered and square integrable representations. The irreducible tempered representations with real central character can be indexed by the irreducible representations of the Weyl group $W$ of type $G_{2}$ (see [BM, p. 34]). Equivalently, these representations can be indexed by the pairs $(n, \rho)$ which appear in the Springer correspondence. The $n$ and $\rho$ will also be elements of the indexing triple for the corresponding tempered representation of $\tilde{H}$. Here $n$ is a nilpotent element of the Lie algebra $\mathfrak{g}=\operatorname{Lie}(G), G$ is the complex simple group over $\mathbb{C}$ of type $G_{2}$ and $\rho$ is an irreducible representation of the component group $Z_{G}(n) / Z_{G}(n)^{\circ}$ (see [Ca]). For each root $\beta \in R$ let $e_{\beta}$ be an element of the root space $\mathfrak{g}_{\beta}$. The five nilpotent orbits in $\mathfrak{g}$ and the corresponding tempered representations of $\tilde{H}$ are as in Table 6.3. The notation $S_{3}$ denotes the symmetric group on three elements, which has irreducible representations indexed by the partitions $(3),(21),\left(1^{3}\right)$ of 3 . We have used the notation of Carter [Ca, p.427] to label the irreducible representations of the Weyl group $W$ of type $G_{2}$.

$\begin{array}{ccccc}\text { Nilpotent orbit } & Z_{G}(n) / Z_{G}(n)^{o} & \text { Indexing triple } & \text { Sq. int. } & W \text { rep. } \\ \text { regular } & 1 & \left(t_{a}, e_{\alpha_{1}}+e_{\alpha_{2}}, 1\right) & \text { yes } & \phi_{1,0} \\ \text { subregular } & S_{3} & \left(t_{e}, e_{\alpha_{1}}+e_{\alpha_{1}+2 \alpha_{2}},(3)\right) & \text { yes } & \phi_{2,1} \\ & & \left(t_{e}, e_{\alpha_{1}}+e_{\alpha_{1}+2 \alpha_{2}},(21)\right) & \text { yes } & \phi_{1,3}{ }^{\prime} \\ \text { subminimal } & 1 & \left(t_{j}, e_{\alpha_{2}}, 1\right) & \text { no } & \phi_{2,2} \\ \text { minimal } & 1 & \left(t_{g}, e_{\alpha_{1}}, 1\right) & \text { no } & \phi_{1,3}{ }^{\prime \prime} \\ 0 & 1 & \left(t_{o}, 0,1\right) & \text { no } & \phi_{1,6}\end{array}$

Table 6.3. Tempered representations and the Springer correspondence 
The only other tempered representations are the representations labeled by the triples $\left(t_{c}, e_{\alpha_{1}}+\right.$ $\left.e_{\alpha_{1}+3 \alpha_{2}}, 1\right)$ and $\left(t_{d}, e_{\alpha_{1}}+e_{\alpha_{1}+2 \alpha_{2}}, 1\right)$. These representations are square integrable but do not have real central character.

The modules labeled by $\left(t_{c}, e_{\alpha_{1}}+e_{\alpha_{1}+3 \alpha_{2}}, 1\right),\left(t_{d}, e_{\alpha_{1}}+e_{\alpha_{1}+2 \alpha_{2}}, 1\right),\left(t_{e}, e_{\alpha_{1}}+e_{\alpha_{1}+2 \alpha_{2}},(3)\right)$, $\left(t_{e}, e_{\alpha_{1}}+e_{\alpha_{1}+2 \alpha_{2}},(21)\right)$ are the ones constructed by Lusztig in [Lu3] 4.20, 4.19, 4.7 and 4.22 respectively. In Lusztig's notation these are the stars (see [Lu3, 4.23]) of the modules labeled by the graphs $\mathcal{G}^{\prime \prime}, \mathcal{G}^{\prime}, \mathcal{G}$ and $\mathcal{G}^{\prime \prime \prime}$, respectively.
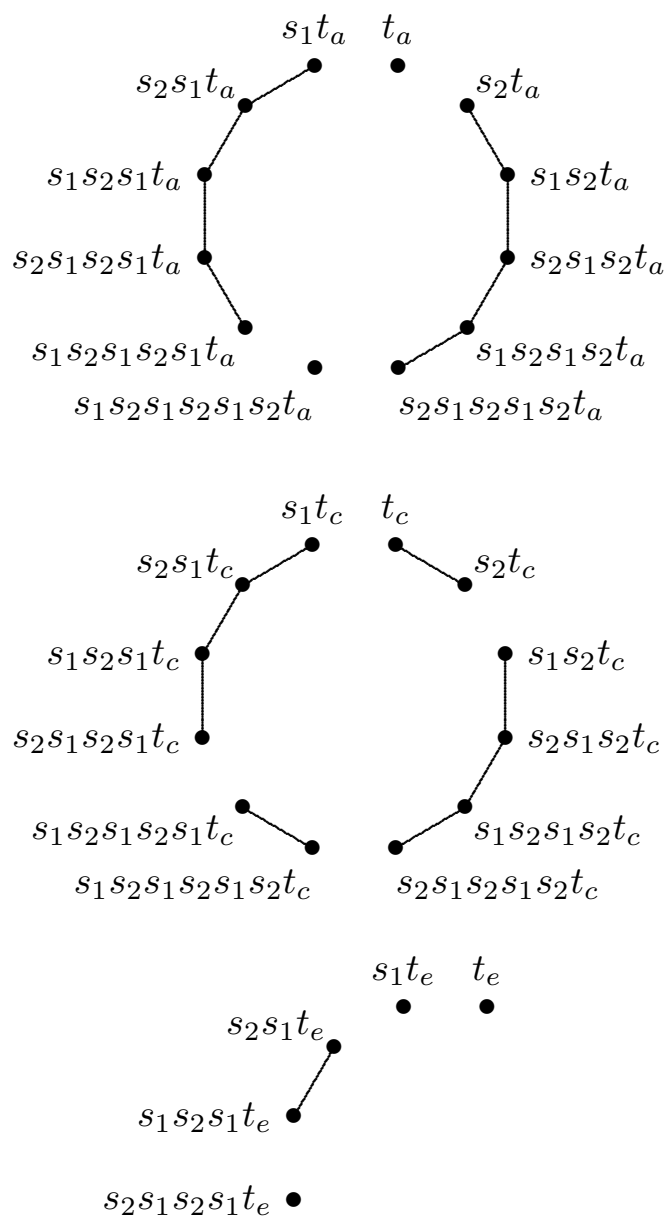

$s_{1} s_{2} s_{1} s_{2} s_{1} t_{e}^{\bullet}$

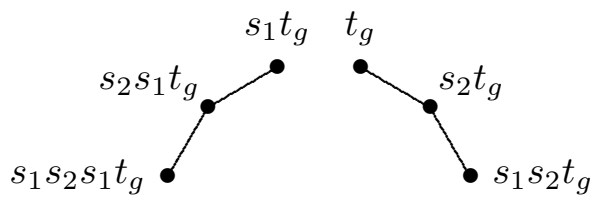

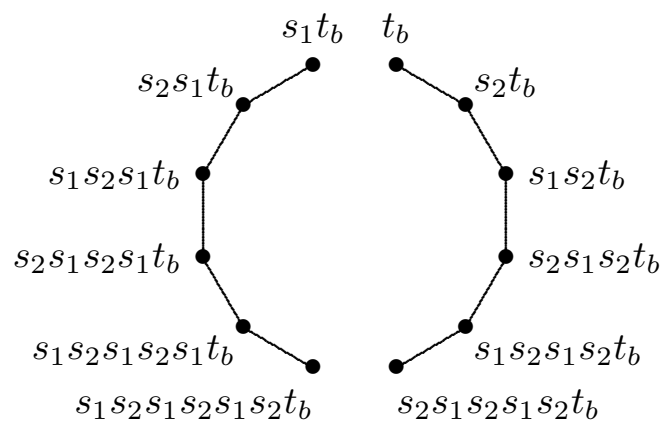
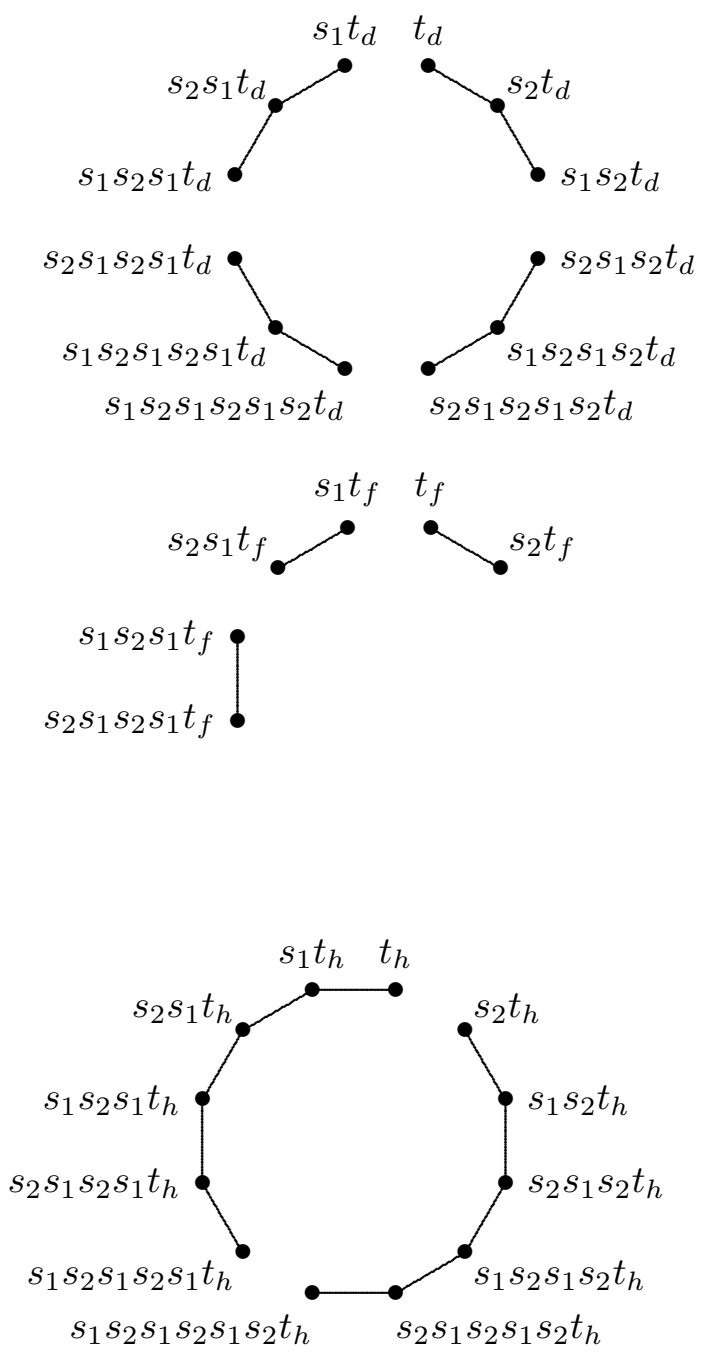

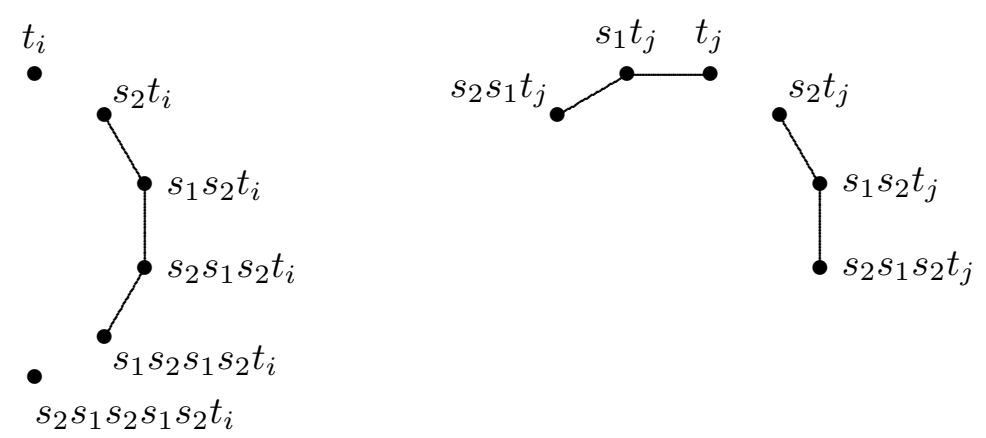

Figure 6.2. Calibration graphs for central characters in Table 6.1

\section{The analysis.}

Central characters $t_{a}, t_{b}, t_{c}, t_{d}, t_{h}$ : The central characters $t_{e}, t_{f}, t_{g}, t_{i}$ and $t_{j}$ are the only ones which have both $Z(t)$ and $P(t)$ nonempty. The other cases are handled by Theorem 1.16 and Theorem 1.11 as in the cases of central characters $t_{a}, t_{b}, t_{g}$ and $t_{o}$ for type $A_{2}$.

The Langlands parameters for each module can be determined from its weight structure. The indexing triple is determined from the Langlands data by using the induction theorem of Kazhdan and Lusztig (see the discussion in [BM, p.34]). Let us give an example to illustrate the procedure. The Langlands parameters $\left(s_{1} s_{2} t_{c},\{1\}\right)$ for the 4 dimensional representation with central character $t_{c}$ correspond to the indexing triple $\left(s_{2} t_{c}, e_{\alpha_{1}}, 1\right)$ which is conjugate to the triple $\left(t_{c}, s_{2} e_{\alpha_{1}}, 1\right)=\left(t_{c}, e_{\alpha_{1}+3 \alpha_{2}}, 1\right)$.

The indexing triples for the tempered representations cannot be determined with the use of the Kazhdan-Lusztig induction theorem. The indexing triples for the tempered representations with real central character are determined from the Springer correspondence, see Table 6.3 and [BM, p.34]. The two tempered representations with central characters $t_{c}$ and $t_{d}$ do not have real central character. By the last two sentences of $[\mathrm{Lu} 3,2.10]$ we know that the indexing triples for these representations contain the subregular nilpotent and that the component groups are isomorphic to $\mathbb{Z} / 3 \mathbb{Z}$ and $\mathbb{Z} / 2 \mathbb{Z}$ respectively. In both cases the component group acts trivially on $K\left(\mathcal{B}_{s, u}\right)$ and so $\rho=1$. The fact that the elements $e_{\alpha_{1}}+e_{\alpha_{1}+3 \alpha_{2}}$ and $e_{\alpha_{1}}+e_{\alpha_{1}+2 \alpha_{2}}$ are representatives of the subregular nilpotent orbit can be derived from the analysis in [Ja, Theorem 4.40] or [Sh]. This determines the triples $\left(t_{c}, e_{\alpha_{1}}+e_{\alpha_{1}+3 \alpha_{2}}, 1\right)$ and $\left(t_{d}, e_{\alpha_{1}}+e_{\alpha_{1}+2 \alpha_{2}}, 1\right)$.

Central character $t_{e}$ : Theorem 1.11 applied to the placed skew shapes $\left(t_{e},\left\{\alpha_{1}, \alpha_{1}+\alpha_{2}\right\}\right),\left(t_{e},\left\{\alpha_{1}\right\}\right)$ and $\left(t_{e},\left\{\alpha_{1}, \alpha_{1}+\alpha_{2}, \alpha_{1}+2 \alpha_{2}\right\}\right)$ produces, respectively, a two dimensional irreducible module $M$ with $\operatorname{supp}(M)=\left\{s_{2} s_{1} t_{e}, s_{1} s_{2} s_{1} t_{e}\right\}$, a one dimensional irreducible module $N$ with $\operatorname{supp}(N)=$ $\left\{s_{1} t_{e}\right\}$ and a one dimensional irreducible module $N^{*}$ with $\operatorname{supp}\left(N^{*}\right)=\left\{s_{2} s_{1} s_{2} s_{1} t_{e}\right\}$. Lusztig [Lu3] Theorem 4.7 constructs a 3 -dimensional irreducible $\tilde{H}$-module $P$ with $\operatorname{dim}\left(P_{t_{e}}^{\text {gen }}\right)=2$ and $\operatorname{dim}\left(P_{s_{1} t_{e}}^{\text {gen }}\right)=1$. In Lusztig's notation this is the module labeled by the graph $\mathcal{G}$ for $\tilde{G}_{2}$.

As described in [Lu3, 4.23] we can twist the module $P$ by an involutive automorphism of $\tilde{H}$ to obtain another 3-dimensional irreducible module $P^{*}$ which has $\operatorname{dim}\left(\left(P^{*}\right)_{s_{2} s_{1} s_{2} s_{1} t_{e}}^{\text {gen }}\right)=2$ and $\operatorname{dim}\left(\left(P^{*}\right)_{s_{1} s_{2} s_{1} s_{2} s_{1} t_{e}}^{\mathrm{gen}}\right)=1$.

All of the modules $M, N, P, N^{*}, P^{*}$ must appear as composition factors of the principal series module $M\left(t_{e}\right)$. By comparing dimensions of weight spaces, any other module $Q$ which appears in a composition series of $M\left(t_{e}\right)$ must have $\operatorname{supp}(Q) \subseteq\left\{s_{2} s_{1} t_{e}, s_{1} s_{2} s_{1} t_{e}\right\}$. Theorem 1.11(b) then implies that $Q$ must be isomorphic to $M$. Thus Theorem 1.15 implies that $M, N, P, N^{*}$, and $P^{*}$ are (up to isomorphism) all the irreducible modules with central character $t_{e}$.

The Langlands parameters for each module are determined from its weight structure. The Kazhdan-Lusztig induction theorem allows us to use the Langlands parameters to determine the 
indexing triples for the modules which are not tempered. Since $t_{e}$ is a real central character the indexing triples for the tempered representations can be determined from the Springer correspondence, see Table 6.3. Alternatively, one can get these triples from [Lu3, 2.10] where it is explained that the nilpotent in the indexing triple is subregular, the variety $\mathcal{B}_{s, u}$ (where $s=t_{e}$ and $u$ is subregular) consists of three disjoint points and a projective line, and the component group is isomorphic to the symmetric group $S_{3}$. The symmetric group $S_{3}$ acts trivially on the line and permutes the three points, which implies that the line corresponds to $\rho=(3)$ (trivial representation of $\left.S_{3}\right)$ and the three points are split between the isotypic components $\rho=(3)$ and $\rho=(21)$. In this case the standard module $M_{s, u,\left(1^{3}\right)}=0$. The projective line in $\mathcal{B}_{s, u}$ corresponds to the two dimensional weight space $\left(P^{*}\right)_{t_{e}}^{\text {gen }}$ in the module $P^{*}$.

Central character $t_{f}$ : Let $\mathbb{C} v_{f}$ and $\mathbb{C} v_{s_{1} f}$ be the one dimensional representations of $\tilde{H}_{\{1\}}$ given by

$$
\begin{array}{ccc}
T_{1} v_{f}=q v_{f}, & X^{\alpha_{1}} v_{f}=q^{2} v_{f}, & X^{3 \alpha_{2}} v_{f}=q^{-2} v_{f}, \\
T_{1} v_{s_{1} f}=-q^{-1} v_{s_{1} f}, & X^{\alpha_{1}} v_{s_{1} f}=q^{-2} v_{s_{1} f}, & X^{3 \alpha_{2}} v_{s_{1} f}=q^{4} v_{s_{1} f} .
\end{array}
$$

Let

$$
M_{1}=\operatorname{Ind}_{\tilde{H}_{\{1\}}^{\tilde{H}}}^{\tilde{\tilde{C}}}\left(\mathbb{C} v_{f}\right) \quad \text { and } \quad M_{2}=\operatorname{Ind}_{\tilde{H}_{\{1\}}^{\tilde{T}}}^{\tilde{\tilde{C}}}\left(\mathbb{C} v_{s_{1} f}\right) .
$$

By Lemma 1.17 these modules have weights $\operatorname{supp}\left(M_{1}\right)=\left\{s_{2} t_{f}, t_{f}, s_{1} t_{f}, s_{2} s_{1} t_{f}\right\}$ and $\operatorname{supp}\left(M_{2}\right)=$ $\left\{s_{1} t_{f}, s_{2} s_{1} t_{f}, s_{1} s_{2} s_{1} t_{f}, s_{2} s_{1} s_{2} s_{1} t_{f}\right\}$ respectively. Both $M_{1}$ and $M_{2}$ are 6 dimensional.

Let $M$ be any $\tilde{H}$-module such that $M_{s_{2} t_{f}} \neq 0$. By Lemma 1.19 and Proposition 1.6, $\operatorname{dim}\left(M_{t_{f}}^{\text {gen }}\right)=\operatorname{dim}\left(M_{s_{2} t_{f}}^{\text {gen }}\right) \geq 2$. Since $\left\langle s_{2} \alpha_{1}, \alpha_{1}^{\vee}\right\rangle=\left\langle\alpha_{1}+3 \alpha_{2}, \alpha_{1}^{\vee}\right\rangle \neq 0$ it follows from Lemma 1.19 (b) that $\operatorname{dim}\left(M_{s_{1} t_{f}}^{\text {gen }}\right) \geq 1$. Then, by Proposition 1.6, $\operatorname{dim}\left(M_{s_{2} s_{1} t_{f}}^{\text {gen }}\right) \geq 1$. Adding these numbers up we see that $\operatorname{dim}(M) \geq 6$. It follows that $M_{1}$ is irreducible. An analogous argument can be applied to conclude that $M_{2}$ is irreducible.

Central character $t_{g}$ : Let $\mathbb{C} v_{g}$ and $\mathbb{C} v_{s_{1} g}$ be the one dimensional representations of $\tilde{H}_{\{1\}}$ given by

Let

$$
\begin{array}{ccc}
T_{1} v_{g}=q v_{g}, & X^{\alpha_{1}} v_{g}=q^{2} v_{g}, & X^{2 \alpha_{2}} v_{g}=q^{-2} v_{g} \\
T_{1} v_{s_{1} g}=-q^{-1} v_{s_{1} g}, & X^{\alpha_{1}} v_{s_{1} g}=q^{-2} v_{s_{1} g}, & X^{2 \alpha_{2}} v_{s_{1} g}=q^{2} v_{s_{1} g}
\end{array}
$$

$$
M_{1}=\operatorname{Ind}_{\tilde{H}_{\{1\}}}^{\tilde{\tilde{H}}}\left(\mathbb{C} v_{g}\right) \quad \text { and } \quad M_{2}=\operatorname{Ind}_{\tilde{H}_{\{1\}}}^{\tilde{\tilde{H}}}\left(\mathbb{C} v_{s_{1} g}\right) .
$$

By Lemma 1.17 these modules have weights $\operatorname{supp}\left(M_{1}\right)=\left\{s_{1} s_{2} t_{g}, s_{2} t_{g}, t_{g}\right\}$ and $\operatorname{supp}\left(M_{2}\right)=$ $\left\{s_{1} t_{g}, s_{2} s_{1} t_{g}, s_{1} s_{2} s_{1} t_{g}\right\}$ respectively. Both $M_{1}$ and $M_{2}$ are 6 dimensional.

Let $M$ be any $\tilde{H}$-module such that $M_{s_{1} s_{2} t_{g}} \neq 0$. By Lemma 1.19 and Proposition 1.6, $\operatorname{dim}\left(M_{t_{g}}^{\mathrm{gen}}\right)=\operatorname{dim}\left(M_{s_{2} t_{g}}^{\mathrm{gen}}\right)=\operatorname{dim}\left(M_{s_{1} s_{2} t_{g}}^{\mathrm{gen}}\right) \geq 2$. Thus $\operatorname{dim}(M) \geq 6$. It follows that $M_{1}$ is irreducible. An analogous argument can be applied to conclude that $M_{2}$ is irreducible.

Central character $t_{i}$ : Let $\mathbb{C} v_{i}$ and $\mathbb{C} v_{s_{2} i}$ be the one dimensional representations of $\tilde{H}_{\{2\}}$ given by

Let

$$
\begin{array}{ccc}
T_{2} v_{i}=q v_{i}, & X^{\alpha_{1}} v_{i}=v_{i}, & X^{\alpha_{2}} v_{i}=q^{2} v_{i}, \\
T_{2} v_{s_{2} i}=-q^{-1} v_{s_{2} i}, & X^{\alpha_{1}} v_{s_{2} i}=q^{6} v_{s_{2} i}, & X^{\alpha_{2}} v_{s_{2} i}=q^{-2} v_{s_{2} i} .
\end{array}
$$

$$
M_{1}=\operatorname{Ind}_{\tilde{H}_{\{2\}}^{\tilde{H}}}^{\tilde{\tilde{C}}}\left(\mathbb{C} v_{i}\right) \quad \text { and } \quad M_{2}=\operatorname{Ind}_{\tilde{H}_{\{2\}}}^{\tilde{H^{\prime}}}\left(\mathbb{C} v_{s_{2} i}\right) .
$$

An argument similar to that for the central character $t_{f}$ shows that $M_{1}$ and $M_{2}$ are irreducible.

Central character $t_{j}$ : Let $\mathbb{C} v_{j}$ and $\mathbb{C} v_{s_{2} j}$ be the one dimensional representations of $\tilde{H}_{\{2\}}$ given by

Let

$$
\begin{gathered}
T_{2} v_{j}=q v_{j}, \quad X^{2 \alpha_{1}} v_{j}=q^{-6} v_{j}, \quad X^{\alpha_{2}} v_{j}=q^{2} v_{j}, \\
T_{2} v_{s_{2} j}=-q^{-1} v_{s_{2} j}, \quad X^{2 \alpha_{1}} v_{s_{2} j}=q^{6} v_{s_{2} j}, \quad X^{\alpha_{2}} v_{s_{2} j}=q^{-2} v_{s_{2} j} .
\end{gathered}
$$

$$
M_{1}=\operatorname{Ind}_{\tilde{H}_{\{2\}}}^{\tilde{H}}\left(\mathbb{C} v_{j}\right) \quad \text { and } \quad M_{2}=\operatorname{Ind}_{\tilde{H}_{\{2\}}}^{\tilde{H}}\left(\mathbb{C} v_{s_{2} j}\right) .
$$

An argument similar to that for the central character $t_{g}$ shows that $M_{1}$ and $M_{2}$ are irreducible. 


\section{REFERENCES}

[BM] D. Barbasch AND A. Moy, A unitarity criterion for p-adic groups, Invent. Math. 98 (1989), 19-37.

[Bou] N. Bourbaki, Groupes et algèbres de Lie, Chapitres 4,5 et 6, Elements de Mathématique, Hermann, Paris 1968.

[Ca] R.W. CARTER, Finite Groups of Lie Type: Conjugacy Classes and Complex Characters, John Wiley and Sons, 1985.

[CG] N. Chriss And V. GinzBurg, Representation Theory and Complex Geometry, Birkhäuser, 1997.

[Ev] S. Evens, The Langlands classification for graded Hecke algebras, Proc. Amer. Math. Soc. 124 (1996), 1285-1290.

[HO1] G.J. Heckman And E.M. Opdam, Yang's system of particles and Hecke algebras, Ann. of Math. (2) 145 (1997), 139-173.

[HO2] G.J. Heckman and E.M. Opdam, Harmonic analysis for affine Hecke algebras, in Current Developments in Mathematics, Intern. Press, Boston, 1996.

[Ja] D. JACKson, On nilpotent orbits of type $G_{2}$, Ph. D. Thesis, University of Sydney, 1997.

[Ka] S-I. KATO, Irreducibility of principal series representations for Hecke algebras of affine type, J. Fac. Sci. Univ. Tokyo Sect. IA Math. 28 (1981), 929-943.

[KL] D. Kazhdan and G. Lusztig, Proof of the Deligne-Langlands conjecture for Hecke algebras, Invent. Math. 87 (1987), 153-215.

[Kr] C. KRILOFF, Some interesting nonspherical tempered representations of graded Hecke algebras, Trans. Amer. Math. Soc., to appear.

[Lu1] G. LuszTig, Singularities, character formulas, and a q-analog of weight multiplicities, Analysis and topology on singular spaces, II, III (Luminy, 1981), Astérisque 101-102, Soc. Math. France, Paris, 1983, 208-229.

[Lu2] G. Lusztig, Affine Hecke algebras and their graded version, J. Amer. Math. Soc. 2 (1989), 599-635.

[Lu3] G. LuszTig, Some examples of square integrable representations of semisimple p-adic groups, Trans. Amer. Math. Soc. 277 (1983), 623-653.

[Lu4] G. LuszTig, Equivariant K-theory and representations of Hecke algebras, Proc. Amer. Math. Soc. 94 (1985), 337-342.

[Ma] H. Matsumoto, Analyse harmonique dans les systèmes de Tits bornologiques de type affine, Lect. Notes in Math. 590, Springer-Verlag, Berlin-New York, 1977.

[Ra1] A. RAM, Calibrated representations of affine Hecke algebras, preprint 1998.

[Ra2] A. RAM, Standard Young tableaux for finite root systems, preprint 1998.

[Ra3] A. RAM, Skew shape representations are irreducible, in preparation.

[RR1] A. RAm AND J. RAmAGGE, Jucys-Murphy elements come from affine Hecke algebras, in preparation. 
[RR2] A. RAm And J. RAmagGe, Calibrated representations and the q-Springer correspondence, in preparation.

[Ro] F. Rodier, Décomposition de la série principale des groupes réductifs p-adiques, Noncommutative harmonic analysis and Lie groups (Marseille, 1980), Lect. Notes in Math. 880, Springer, Berlin-New York, 1981, 408-424.

[St] R. Steinberg, Endomorphisms of linear algebraic groups, Mem. Amer. Math. Soc. 80, Amer. Math. Soc., Providence, R.I. 1968.

[Sh] U. StuhleR, Unipotente und nilpotente Klassen in einfachen Gruppen und Liealgebren vom typ $G_{2}$, Indag. Math. 74 (1971), 365-378.

[Xi] N. XI, Representations of affine Hecke algebras, Lect. Notes in Math. 1587, Springer-Verlag, Berlin, 1994. 